ORDINES MILITARES

COLLOQUIA TORUNENSIA HISTORICA

Yearbook for the Study of the Military Orders

ISSN (print) 0867-2008 / ISSN (online) 2391-7512

DOI: http://dx.doi.org/10.12775/OM.2016.010

pp. $189-220$

RENGER E. DE BRUIN

Centraal Museum

Agnietenstraat 1

NL-3512 XA Utrecht

Netherlands

rbruin@centraalmuseum.nl

\title{
EINE GELUNGENE NEUORDNUNG. DIE BALLEI UTRECHT DES DEUTSCHEN ORDENS, $1753-1795$
}

\section{KeYwORDS}

Military Orders; Teutonic Order; order's reform; Bailiwick of Utrecht; Early Modern Period; Netherlands in I 8 th century

A m 25. September 1753 kam das Kapitel der Ballei Utrecht im Deutschhaus in Utrecht zusammen. Die Versammlung war von Frederik Willem Baron Torck (I69 I-I 76I), dem Komtur von Dieren, einberufen worden, und wurde von ihm auch eröffnet. Die Ballei hatte zu diesem Zeitpunkt keinen Landkomtur und auch keinen Koadjutor. Die letzten Amtsinhaber waren einige Jahre zuvor verstorben, ohne dass Nachfolger benannt worden waren. Dieser Umstand ist bezeichnend für das Chaos, in dem die Ballei Mitte des i 8. Jahrhunderts verkehrte. Baron Torck und Unico Wilhelm Graaf van Wassenaer van Twickel (1692-1 766), die während der viertägigen Versammlung im September I 753 zum Landkomtur bzw. Koadjutor gewählt wurden, waren der Ansicht, dass es so nicht weitergehen konnte. Van Wassenaer machte Vorschläge tot redres der vervallene saken (,zur Wiederherstellung der verfallenen Sachen“) und erhielt den Auftrag, diese im Rahmen einer Kommission auszuarbeiten. ${ }^{1}$

1 Archief Ridderlijke Duitsche Orde Balije van Utrecht, Oud Archief (weiter: ARDOU-OU), Inv. Nr. 11, Resolutiën van de landcommanderij van Utrecht (1561-1827), Band 3, Fol. 96r.

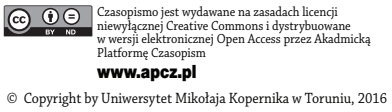


In diesem Beitrag möchte ich aufzeigen, wie die Ballei Utrecht nach dem Bruch mit dem Hochmeister in Verfall geriet, welche Lösungsvorschläge van Wassenaer antrug, wie diese umgesetzt wurden, und zu welchen Ergebnissen sie führten. Die Studie zur Neuordnung der Ballei Utrecht ist Teil einer umfangreicheren Forschungsarbeit zur Entwicklung der Ballei in der zweiten Hälfte des i 8. und den ersten Jahrzehnten des I9. Jahrhunderts. Im Fokus standen hierbei die Aufhebung durch Napoleon im Jahr I 8 I I und die anschließende Wiedererrichtung durch den niederländischen König Wilhelm I. im Jahr I 8 I 5 . Die Ergebnisse dieser Arbeit wurden $20 \mathrm{I} 2$ in einer niederländischen Monographie veröffentlicht. ${ }^{2}$ Die Entwicklungen rund um die Aufhebung wurden in den Teilen V und VI der englischsprachigen Serie The Military Orders publiziert. ${ }^{3}$ Der vorliegende Beitrag macht einen weiteren Teil der Arbeit für die internationale Forschung zugänglich.

\section{Der Niedergang EINER Institution}

Die Krise, aus der van Wassenaer und seine Kollegen einen Ausweg zu finden versuchten, umfasste mehrere Aspekte des Ordenswesens: Die Nachfolge im Amt des Landkomturs, die Auswahl der Mitglieder, die Verwaltung der Güter und die Buchhaltung. Akut geworden war die Krise mit dem Tod des Landkomturs Hendrik van Isselmuden (1680-175 I), für den kein Nachfolger benannt worden war. Die Ursache des Niedergangs lag jedoch viel weiter zurück und war struktureller Natur. Nach ihrem schnellen Wachstum im I 3. und I 4. Jahrhundert bekam die Ballei Utrecht im Verlauf des I 5 . Jahrhundert die Malaise des Deutschen Ordens zu spüren, die mit der Schlacht bei Tannenberg im Jahr I 4 IO eingesetzt hatte. Darüber hinaus wurde die Ballei in die politischen Ereignisse rund um das Stift Utrecht verwickelt, die schließlich zu dessen Angliederung an das burgundische Erbe Karls V. im Jahr I 528 führten. In diesem Zeitraum von über einem Jahrhundert gab es einige Aufschwünge, etwa um i 475 unter Johan van Drongelen, doch die Grundtendenz war negativ. ${ }^{4}$

2 R. E. de Bruin, Bedreigd door Napoleon. De Ridderlijke Duitsche Orde, Balije van Utrecht 1753-1838, Hilversum 2012.

3 Ders., Hidden in the Bushes: The Teutonic Order of the Bailiwick of Utrecht in the 1780-1806 Revolutionary Period, in: The Military Orders, vol. V: Politics and Power, ed. P. Edbury, Farnham 2012, S. 349-361; ders., The narrow escape of the Teutonic Order Bailiwick of Utrecht, 1811-1815, in: The Military Orders, vol. VI, p. 2: Culture and Conflict in Western and Northern Europe, ed. Jochen Schenk, Mike Carr, London-New York 2017, S. 222-232.

4 J. A. Mol, The 'Hospice of the German Nobility': Changes in the Admission Policy of the Teutonic Knights in the Fifteenth Century, in: Mendicants, Military Orders and Regionalism in Medieval Europe, hrsg. v. J. Sarnowsky, Aldershot 1999, S. 115-130; ders., Crisis in Pruisen, crisis in de 
Deutlich ernster wurde die Situation mit dem Aufstand der niederländischen Provinzen gegen Karls Sohn Philipp II. Da die Provinzen Holland und Seeland das spanische Joch bereits abgeschüttelt hatten, während der Hauptsitz noch in königstreuem Gebiet lag, war der Landkomtur von wichtigen Kommenden wie Maasland und Leiden abgeschnitten. Diese Teilung wurde beendet, als sich im Jahr I 576 alle niederländischen Provinzen gegen die spanische Herrschaft vereinten. Der sich jahrzehntelang hinziehende Kampf gegen die spanischen Truppen stellte jedoch eine schwere Belastung dar. Die katholische Identität der Ballei und die Verbindung zum Hochmeister kamen in Gefahr. Von I 579 an bemühte sich Landkomtur Jacob Taets van Amerongen (I 542-I6 I 2), die Ballei neu zu beleben. Er strebte danach, ihr ihren katholischen Charakter zurückzugeben und die unter seinen Vorgängern vernachlässigte Disziplin wiederherzustellen. Er sorgte dafür, dass die Gottesdienste wieder ordnungsgemäß verliefen und aufgegebene Traditionen wie die Fußwaschung am Gründonnerstag wieder aufgenommen wurden. Um die Kontinuität der Ballei zu unterstreichen, gab er die Fertigung einer Portraitreihe aller Landkomture ab I 23 I in Auftrag.'

In seinem Streben nach Erhalt der katholischen Identität setzte er sich dem politischen Trend diametral entgegen. Wie zuvor bereits die Provinz Holland verboten im Frühjahr I 580 auch die Stadt und Provinz Utrecht die Ausübung des katholischen Gottesdienstes. Die Machthaber in Utrecht sympathisierten allerdings mit einer gemäßigten Form des Protestantismus und nahmen außerdem - auch im eigenen Sinne - Rücksicht auf etablierte Interessen. Sie hoben die fünf Kapitel daher nicht auf, sondern ließen sie in säkularisierter Form fortbestehen. Taets van Amerongen machte von diesem Spielraum erfolgreich Gebrauch. Er wusste die Verbindung zum Hochmeister zu erhalten und mit Hilfe des Landkomturs von Biesen die Statutenänderung von 1606 umzusetzen. Es gelang ihm sogar, vor seinem Tod im Jahr I 6 I 2 noch einen katholischen Nachfolger zu präsentieren. Doch der Versuch, die Identität der Ballei Utrecht zu erhalten, war zum Scheitern verurteilt. Im Jahr I 6 I 8 kam in der Provinz eine Gruppe strenger Calvinisten an die Macht. Und auch die internationale Konstellation war ungünstig. $\mathrm{Zu}$ einem Zeitpunkt, zu dem im Deutschen Reich der (Dreißigjährige) Krieg ausbrach und - nach dem Auslaufen des I 609 geschlossenen Waffenstillstands - das Wiederaufflammen des niederländischen Kampfes gegen die Spanier drohte, war der Fortbestand einer dem Habsburger Hochmeister treuen katholischen Institution

balijen? De casus Utrecht, 1443-1469, in: Vechten, bidden en verplegen. Opstellen over de ridderorden in de Noordelijke Nederlanden, hrsg. v. J. A. Mol, Hilversum 2011, S. 206-222.

5 D. Meuwissen, Gekoesterde traditie. De portretreeks met de landcommandeurs van de Utrechtse Balije van de Ridderlijke Duitsche Orde, Hilversum 2011, S. 91-107. 
gänzlich unerwünscht. Die Stände von Utrecht, die seit der Absetzung Philipps II. als Landesherren dessen Rolle als Souverän der Ballei Utrecht übernommen hatten, setzten 16 I9 die Ernennung eines nicht katholischen Landkomturs durch. Dessen Nachfolger wiederum wurde ein überzeugter Calvinist: Hendrik Casimir I. von Nassau-Dietz (1612-1640), der mit dem Statthalter Moritz (1567-1625), dem militärischen Führer des Aufstands, verwandt war. In den Jahren I 637-1640 wurde die Protestantisierung durch die Abschaffung des Zölibats abgeschlossen, was zum Bruch mit dem Hochmeister führte. ${ }^{6}$ Dieser ließ den Platz Utrechts im Kapitelsaal unbesetzt. Es folgten diverse Versuche zur Wiedervereinigung, die jedoch erfolglos blieben. Nicht der reformierte Charakter war das unüberwindbare Problem, sondern der Widerstand der Utrechter Stände einerseits und die Zölibatsforderung des Hochmeisters andererseits. ${ }^{7}$

Seit dem Bruch wurden die Utrechter Komturämter durch verheiratete Adlige protestantischen Glaubens besetzt. Wer als Katholik bereits Mitglied war, durfte bleiben. Wer neu beitreten wollte, musste jedoch der vorherrschenden reformierten Kirche angehören. Damit stellte die Ballei Utrecht sich auf eine Linie mit den Ritterschaften und den Stadträten, die diese Regel bereits einige Jahrzehnte zuvor eingeführt hatten. ${ }^{8}$ Die Ballei war nun Teil des politischen Systems der neuen Republik der Vereinigten Niederlande, die I 648 mit dem Westfälischen Frieden international anerkannt wurde. Die Ordensmitglieder waren als Abgeordnete in den politischen Organen oder als Militäroffiziere aktiv ins System des jungen Staates eingebunden. Die Mitgliedschaft war Angehörigen niederländischer (und deutscher) Adelsfamilien vorbehalten. Zukünftige Mitglieder, die sogenannten Expektanten, wurden im Kindesalter von den amtierenden Komturen angemeldet. Wenn sie an der Reihe waren, mussten sie den Nachweis über vier adelige

6 J. A. Mol, Trying to survive. The Military Orders in Utrecht, 1580-1620, in: The Military Orders and the Reformation. Choices, State Building and the Weight of Tradition, hrsg. v. J. A. Mol, K. Militzer, H. Nicholson, Hilversum 2006, S. 181-208; D. Grögor-Schiemann, Die Deutschordensballei Utrecht während der Reformationszeit: die Landkommende zwischen Rebellion und Staatsbildung (Quellen und Studien zur Geschichte des Deutschen Ordens 68), Weimar 2015, S. $180-199$.

7800 Jahre Deutscher Orden. Ausstellung des Germanischen Nationalmuseums Nürnberg in Zusammenarbeit mit der Internationalen Historischen Kommission zur Erforschung des Deutschen Ordens, Germanisches Nationalmuseum, 30.6.-30.9.1990, hrsg. v. Germanischen Nationalmuseum, red. v. U. Arnold, G. Bott, Gütersloh-München 1990, S. 252, 256; J. H. de Vey Mestdagh, De Utrechtse Balije der Duitse Orde. Ruim 750 jaar geschiedenis van de Orde in de Nederlanden, Utrecht-Bilzen 1989, S. 48-53.

8 J. L. Price, Culture and Society in the Dutch Republic during the 17th Century, London 1974, S. 36; J. I. Israel, The Dutch Republic. Its Rise, Greatness and Fall, 1477-1806, Oxford 1995, S. 426-432. 
Großelternteile und das reformierte Glaubensbekenntnis erbringen. Daraufhin konnten Sie gegen Zahlung eines rustgeld, einer Aufnahmegebühr, als jonkheer beitreten, um beim Freiwerden eines Sitzes im Kapitel als Komtur eingesetzt zu werden. Diese Regeln basierten auf Beschlüssen der Utrechter Stände aus den Jahren 1615, 1619, 1637 und 1640. Durch sie war die Ballei in das niederländische Staatssystem integriert. Das war jedoch problematisch, da es hierfür keinen institutionellen Rahmen gab: Über die nach wie vor geltenden Statuten aus dem Jahr I 606, die die deutlich katholische Handschrift des Hochmeisters trugen, war die Ballei formal noch an das mittelalterliche Ordenssystem gebunden. ${ }^{9}$

Dieser hybride Charakter wirkte sich vor allem bei der Verwaltung der Besitztümer negativ aus. Die Mitgliedschaft im Deutschen Orden war immer mehr zur Nebenaufgabe geworden. Die verheirateten Komture blieben in ihren Schlössern oder Stadthäusern wohnen. Die Komturhäuser wurden überflüssig und gerieten in Verfall. Sie wurden verkauft oder abgerissen. Die Komture leiteten ihre Kommenden aus der Ferne, und da ein Nachrücksystem bestand, gab es keine Kontinuität. Einer der wenigen, die ihre Aufgaben noch ernst nahmen, war Frans Steven Carel van Randwijck (1697-1785). Dies geht aus seiner Korrespondenz mit den Verwaltern der nacheinander von ihm geleiteten Kommenden Doesburg, Schoten und Maasland hervor. ${ }^{10}$ Doch er war eine Ausnahme, und seine Umzüge von einer Kommende zur nächsten zeigen, dass auch die Amtszeit eines aktiven Komturs nur wenige Jahre dauerte. Von den Landkomturen beschäftigten sich nur wenige intensiv mit der Ballei. Die friesischen Statthalter, die im 17 . Jahrhundert das Amt des Landkomturs bekleideten, sahen hierin nicht mehr als einen lukrativen $\mathrm{Ne}-$ benverdienst. Eine Ausnahme war Godard van Reede van Athlone (1644-1703), der das Amt von 1697 bis 1703 innehatte. Als hoher Offizier unter König-Statthalter Wilhelm III. hatte er für diesen in Irland ruhmreiche Siege erlangt. Hierfür war ihm ein irischer Grafentitel verliehen worden. Als er die höchste Position in der Ballei Utrecht erreicht hatte, bemühte er sich nach Kräften, die Institution zu neuem Leben zu erwecken - unter anderem, indem er das Kapitel wieder häufiger einberief. Auch sorgte er dafür, dass die zwischenzeitlich unterbrochene Portraitserie fortgeführt wurde. ${ }^{11}$ Seine Nachfolger dagegen waren wesentlich weniger engagiert, und als es nach dem Tod Hendrik van Isselmudens im Jahr I75 I zu einem Interregnum kam, war das Chaos komplett.

9 de Vey Mestdagh (wie Anm. 7), S. 44; de Bruin, Bedreigd door Napoleon (wie Anm. 2) S. 67-71.

10 Gelders Archief (weiter: GA) 609, Archief van de familie Van Randwijck, 1292-1870, Inv. Nr. 218 l, Brieven van F. S. C. van Randwijck als commandeur van Schoten, 1 omslag; Inv. Nr. 218 b, Brieven van F. S. C. van Randwijck als commandeur van Rhenen; Inv. Nr. 219, Stukken betreffende het beheer van de commanderij van Maasland.

11 Meuwissen (wie Anm. 5), 146-156. 


\section{Die Reformvorschläge Unico Wilhelm Van Wassenaers}

Dies war die Situation, in der Frederik Willem Torck im September 1753 eine Kapitelversammlung anberaumte. Indem er selbst zum Landkomtur und Unico Wilhelm van Wassenaer zum Koadjutor gewählt wurde, verfügte die Ballei wieder über eine Führung. Es war der neue Koadjutor, der bereits zu Beginn der Versammlung den Niedergang und seine Ursachen zur Sprache brachte. Seit 1733 Mitglied des Kapitels, war van Wassenaer bereits über 6o, als er ins zweithöchste Amt der Ballei berufen wurde. Er entstammte dem ältesten noch existierenden Adelsgeschlecht der Provinz Holland und war extrem reich. Seine umfangreichen Besitztümer lagen verteilt über die Provinzen Holland, Overijssel und Friesland. Er hatte seinem Land als Diplomat gedient und den Ständen von Overijssel angehört, und war zu diesem Zeitpunkt noch Mitglied der Stände Hollands. Während einer Mission, deren Ziel es war, das Erzbistum Köln aus dem Österreichischen Erbfolgekrieg herauszuhalten, war er mit dem Hochmeister Clemens August von Wittelsbach (1700-176I) in Kontakt gekommen, der gleichzeitig Erzbischof von Köln war. Van Wassenaer durfte einer Inauguration von Ordensrittern beiwohnen und war hiervon sehr beeindruckt. Anschließend ließ er sich vom Kölner Hofmaler George de Marées (1697-1776) selbst mit Ordensmantel und Kreuz portraitieren. ${ }^{12}$

Als Diplomat war er nicht sonderlich erfolgreich, und nachdem Statthalter Wilhelm IV. (I7II-I75I) an die Macht gekommen war, wurde er auch politisch ins Abseits gedrängt. Mit umso mehr Elan widmete er sich der Verwaltung seiner Landgüter, der Kultur - er war ein großer Musikliebhaber - und dem Deutschen Orden.

Beim Orden durfte er seine Ideen tot redres van de vervallene saken en verbetering der administratie („zur Wiederherstellung der verfallenen Sachen und Verbesserung der Verwaltung") in einer Kommission ausarbeiten, der auch der vorgenannte Frans Steven Carel van Randwijck und der Komtur von Rhenen, Jasper Hendrik van Lynden van Ressen (1693-1759), angehörten. ${ }^{13}$ Unter der entschlossenen Führung des Koadjutors stellten sie im Sommer 1754 einen I 5 -Punkte-Plan fertig, der konkrete Empfehlungen zur Lösung der dringendsten

12 L. J. van der Klooster, Unico Wilhelm van Wassenaer in zijn culturele milieu, in: Unico Wilhelm van Wassenaer 1692-1766, componist en staatsman, hrsg. v. R. Rasch, K. Vlaardingerbroek, Zutphen 1993, S. 116-117.

13 ARDOU-OU, Inv. Nr. 11-3, Fol. 96. 
organisatorischen, finanziellen und administrativen Probleme umfasste. ${ }^{14}$ Dieser Plan wurde während der anschließenden Entscheidungsprozesse immer detaillierter ausgearbeitet, doch die Grundstruktur stand bereits im Sommer 1754 fest. Um ein erneutes Interregnum zu vermeiden, schlug die Kommission vor, beim Tod eines Landkomturs den Koadjutor, und falls dieses Amt nicht besetzt war, den ältesten Komtur als seinen Nachfolger einzusetzen. Das Nachrücksystem wurde hiermit eindeutig festgeschrieben. Die Komture sollten ein festes Gehalt beziehen, das sich nach ihrem Rang richtete. Wer eine Position höher rückte, sollte auch ein höheres Gehalt bekommen. Auch die Anmeldung von Expektanten sollte ordentlicher verlaufen. Hierzu sollte die Kontrolle der Nachweise über die adlige Abstammung und das reformierte Glaubensbekenntnis verbessert werden.

Die neuen Regeln stellten einen Bruch mit der Vergangenheit dar, in der die Komture die lokalen Abteilungen der Ballei verwaltet hatten. Die hybride Struktur, die durch die Veränderungen in den Jahren I 6 19-1640 entstanden war, wurde jetzt durch ein transparentes System ersetzt. Die Verwaltung der Güter sollte nicht mehr in der Verantwortung der Komture liegen, sondern zentral vom Deutschhaus in Utrecht aus erfolgen, und zwar durch einen festangestellten Generalverwalter, der hierfür ein redelijk salaris (,angemessenes Gehalt") beziehen sollte. ${ }^{15}$ Der Kandidat, den die Kommissionsmitglieder hierfür im Auge hatten, war der Verwalter der Landkommende, Gijsbert Dirk Cazius (1722-1804). Die Zusammenarbeit mit ihm bei der Ausarbeitung ihrer Vorschläge war sehr harmonisch verlaufen. Das Gegenteil war der Fall, was den Sekretär Adriaan van Bronckhorst (1724- I 806) betraf. Dieser versuchte mit allen Mitteln, van Wassenaer und die Seinen zu behindern. Van Randwijck musste sogar eine Art Einbruch begehen, um an die notwendigen Urkunden zu kommen. Ziel der zentralisierten Güterverwaltung durch einen Generalverwalter in der Landkommende war es, einen Überblick über die Einnahmen und Ausgaben zu erhalten. Van Wassenaer hatte auf der Kapitelversammlung im September 1753 erklärt, dass die Immobilien des Ordens entweder gut bewirtschaftet oder abgestoßen werden sollten. Bei der Bewirtschaftung war vor allem das regelmäßige und vollständige Kassieren der Pachten wichtig. Die Ballei verfügte aufgrund von Schenkungen aus dem Mittelalter noch über einen umfangreichen Besitz an Landwirtschaftsflächen, die jedoch sehr verstreut lagen. Pachtbauern hatten die chaotische Verwaltung ausgenutzt und Pachten nicht in voller Höhe oder gar nicht bezahlt. Naturkatastrophen wie Überschwemmungen

\footnotetext{
14 ARDOU-OU, Inv. Nr. 189, Rapport uitgebracht aan het kapittel van de D.O. door de coadjutor en twee commandeurs over de vervallen staat der landcommanderij, met voorstellen tot verbetering van die toestand (1754) fol. 7.

15 ARDOU-OU, Inv. Nr. 11-3, Fol. 96.
} 
und Ausbrüche von Viehseuchen hatten ihnen dabei als Entschuldigung gedient. ${ }^{16}$ Für das Gelingen des neuen Verwaltungssystems war eine gute Zusammenarbeit zwischen Generalverwalter und Landkomtur unabdingbar. Letzterer erhielt im Vergleich zur bisherigen Situation viel mehr Befugnisse, da die Kommenden nun nicht mehr von den jeweiligen Komturen, sondern zentral von der Landkommende aus verwaltet wurden. Hierzu war die ständige Rücksprache zwischen Generalverwalter und Landkomtur erforderlich. Die durch den Generalverwalter zu führende Buchhaltung sollte regelmäßig durch den Landkomtur kontrolliert und alle paar Jahre dem vollzähligen Kapitel vorgelegt werden. In den dazwischen liegenden Jahren sollte eine aus mehreren Komturen und dem Landkomtur bestehende Kommission die Finanzen begutachten. Für eine gute Verwaltung war darüber hinaus eine ordentliche Archivierung erforderlich.

\section{Die Annahme und Umsetzung Der Reformvorschläge}

Die Vorschläge der Kommission unter van Wassenaer kamen in der nächstfolgenden Kapitelversammlung im September 1756 auf den Tisch. Die Kommission konnte auf die volle Unterstützung durch Landkomtur Torck zählen. Die Argumente waren deutlich: Das verwalterische Chaos hatte zu großen Defiziten geführt, die sich in Folge von Überschwemmungen und Viehseuchen weiter vergrößert hatten. Aufgrund von unklaren Aufgabenbeschreibungen waren Konflikte entstanden, und durch das Fehlen klarer Nachfolgeregeln war es zum Interregnum von 1751-1753 gekommen. Hierdurch hatten sich die bestehenden Probleme noch deutlich verschärft. Obwohl die Komture die Entscheidungsgewalt über die eigene Kommende abgeben mussten, war die Aussicht auf ein festes, dem Rang entsprechendes Gehalt derart attraktiv, dass die Versammlung sich ohne Murren einverstanden erklärte. Der Vorschlag wurde "geaccepteerd en gearresteerd“ (angenommen und beschlossen). ${ }^{17}$ Der nächste Schritt bestand darin, den Plan den Utrechter Ständen vorzulegen, die als Souverän des Ordens ihre Zustimmung erklären mussten, bevor die Umstrukturierung umgesetzt werden konnte. Dieser Schritt erfolgte umgehend: Noch am selben Tag, dem 22. September 1756, führte die Kommission unter van Wassenaer ein Gespräch mit einer Delegation

16 J. A. Faber, Cattle Plague in the Netherlands during the Eigteenth Century, Mededelingen van de Landbouwhogeschool te Wageningen 62 (1962), S. 1-7; R. N. J. Rommes, 'Geen vrolyk geloei der melkzwaare koeien.' Runderpest in Utrecht in de achttiende eeuw, Jaarboek Oud-Utrecht (2001), S. 87-136. J. de Vries, A. van der Woude, The First Modern Economy: Success, Failure, and Perseverance of the Dutch Economy, 1500-1815, Cambridge 1997, S. 258-259.

17 ARDOU-OU, Inv. Nr. 11-3, Fol. 98. 
aus hochrangigen Ständemitgliedern unter Leitung des Präsidenten der Utrechter Ständeversammlung. Dieser unterstrich nochmals, dass die Stände von Utrecht an die Stelle des Hochmeisters und des Landesherren getreten waren und damit die Instanz waren, der gegenüber die Ballei verantwortlich war. Die Gespräche verliefen nicht ohne Probleme. Die Ständedelegation warf der Ballei Schlampigkeit bei der Zulassung von Mitgliedern und unzureichende Rücksprache mit dem Souverän beim Verkauf von Gütern vor. Auch wurde der Vorwurf geäußert, dass die Ballei eigenmächtig eine Statutenänderung umgesetzt habe, was jedoch scharf zurückgewiesen wurde. Den Komturen zufolge handelte es sich lediglich um den Beschluss, die Ordenskreuze so zu tragen wie es in „de andere Duijtschen Balijen“ üblich ist. ${ }^{18}$ Dies schien ein nachrangiger Punkt zu sein, doch für die Stände von Utrecht war er von Bedeutung. Sie hatten sich als Souverän stets gegen die Annäherung an den Hochmeister gewehrt, da sie keine ausländische Kontrolle über die Ballei Utrecht wünschten. Der Konflikt eskalierte, und die Gespräche wurden abgebrochen. Schließlich gab die Ballei Utrecht hinsichtlich der Ordenskreuze nach. Daraufhin erhielt sie die Genehmigung zur Umsetzung der Reformen. Die Ständeversammlung beauftragte die eigene Exekutive, die Gedeputeerde Staten, die Angelegenheit abzuhandeln und die Ballei Utrecht entsprechend zu informieren. ${ }^{19}$

Das Genehmigungsschreiben der Utrechter Stände wurde auf der folgenden Kapitelversammlung besprochen. Diese fand allerdings erst vier Jahre später statt, von 24. bis 27. September 1760. Die Komture kamen unter Leitung von Landkomtur Torck im Deutschhaus in Utrecht zusammen. Sie reagierten auf den detaillierten Bericht, den die Kommission unter van Wassenaer zu den Reformplänen erstellt hatte. Der Koadjutor verteidigte erneut die Konzentration der gesamten Güterverwaltung bei der Landkommende, unter Verweis auf de veelvuldige inconvenienten van de particuliere beheeringe der commanduijrijen, en de noodsakelikheid als mede de bekwaamste middelen omme daar in ten gemeene voordeelen, redres te maken („die vielfältigen Inkonvenienzen der privaten Verwaltung der Kommenden, und die Notwendigkeit ebenso wie die geeignetsten Mittel, diese zum allgemeinen Vorteil zu reorganisieren"). Anschließend betonte van Wassenaer nochmals, „welke voordeelen de leeden van het capittul daarbij souden genieten" (welche Vorteile die Mitglieder des Kapitels dabei genießen würden): Sie bräuchten sich nicht mehr um die Verwaltung der Kommenden zu kümmern,

18 Het Utrechts Archief (weiter: HUA) 233, Archief van de Staten van Utrecht, Inv. Nr. 234, Derde serie van de resoluties (1673-1795) 84, 22.09.1756.

19 HUA 233, Inv. Nr. 234-84, 12.10.1756. Zur inneren Struktur der Utrechter Stände siehe R. E. de Bruin We and Our Successors Shall Do Justice by All. Provincial Government in Utrecht: A Historical Perspetive, Utrecht 2003, S. 58-78. 
würden aber gleichzeitig ein garantiertes Einkommen beziehen. Das muss die Herren überzeugt haben. Leider sind die Reaktionen der Mitglieder nicht in den Protokollen dokumentiert. Es ist lediglich vermerkt, dass es Diskussionen gab, und dass die Vorschläge na rijpe deliberatie [...] met eenparig genoegen en toestemming goedgevonden en geresolveert („,nach eingehender Erörterung [...] einvernehmlich angenommen und beschlossen") wurden. ${ }^{20}$

Der Beschluss besagte, dass mit Wirkung vom I. Januar I 762 alle Kommenden in die Zuständigkeit des Generalverwalters fielen. Mit dem Amt des Generalverwalters wurde wie geplant Gijsbert Dirk Cazius betraut. Er sollte die Generalkasse verwalten und eine ordentliche Buchhaltung führen. Alle Einnahmen sollten in die Generalkasse fließen, aus der auch die Ausgaben zu begleichen waren - die Kosten für die Instandhaltung der Besitztümer, die Steuern, die Gehälter der Komture und des Personals, und einige kleine Ausgaben wie Beiträge zu Pastorengehältern und Zahlungen an Diakonien. Letztere Posten, die nur wenige Prozent der Gesamtausgaben ausmachten, bildeten das kümmerliche Überbleibsel der karitativen Aktivitäten des Ordens. Der Kostenanteil für die Gehälter der Komture war viele Male größer. Die Jahresgehälter waren gestaffelt von 60 Gulden für den Komtur von Schoonhoven, der den niedrigsten Rang hatte, bis 5500 Gulden für den Landkomtur. Eine Umrechnung dieser Beträge in Euro ist äußerst schwierig. Zum Vergleich: Für die Mitgliedschaft in der Ständeversammlung seiner Provinz erhielt ein Adliger um die 5000 Gulden pro Jahr. ${ }^{21}$ Ein Adliger wie Unico Wilhelm von Wassenaer, der äußerst vermögend war und zahlreiche Funktionen innehatte, konnte aus seinem Vermögen und seinen Ämtern ein Einkommen von rund 20000 Gulden pro Jahr erzielen. Machte das Koadjutorengehalt von 5000 Gulden in seinem Fall schon einen großen Teil seines Gesamteinkommens aus, so stellte es für Adlige mit kleinerem Familienkapital erst recht ein attraktives Einkommen dar. Neben dem festen Gehalt konnten die Komture auch noch Zulagen erhalten, wenn Überschüsse zu verteilen waren.

Angesichts derartiger finanzieller Aussichten, die zudem noch mit einer Verringerung der Aufgaben verbunden waren, ist es kein Wunder, dass die Kapitelmitglieder Befugnisse an den Landkomtur und den Generalverwalter abtraten. Letzterer hatte die Aufgabe, den groten omslag („die große Reorganisation“) durchzuführen. Dass er hierfür ein beträchtliches Gehalt beziehen sollte, wurde als angemessen erachtet. Cazius erhielt vom Koadjutor Instruktionen, an die er

20 ARDOU-OU, Inv. Nr. 11-3, Fol. 110v.

21 J. Aalbers, Geboorte en geld. Adel in Gelderland, Utrecht en Holland tijdens de eerste helft van de achttiende eeuw, in: De bloem der natie. Adel en patriciaat in de Noordelijke Nederlanden, hrsg. v. J. Aalbers, M. Prak, Meppel 1987, S. 73. 
sich zu halten hatte. Hierin war auch die Aufgabenverteilung zwischen Generalverwalter und Sekretär geregelt. Van Wassenaer und Cazius hatten dabei das Glück, dass Sekretär Adriaan van Bronckhorst aus eigenen Stücken abtrat, da er Mitglied des Utrechter Stadtrats geworden war. Van Wassenaer schrieb erleichtert an van Randwijck: Nous voila heuresement debarrassé du secretaire avec honneur. ${ }^{22}$ Zum Nachfolger ernannte er im Anschluss an ein offenes Bewerbungsverfahren Frederik Sigismund Coenraad van Nes ( 1727-I 805). Zwischen ihm und dem Verwalter entwickelte sich eine fruchtbare Zusammenarbeit.

Nach der Zustimmung des Kapitels musste der Beschluss noch durch die Utrechter Stände genehmigt werden. Dies geschah bereits wenige Tage später. Sieben Jahre nach der analytischen Ansprache van Wassenaers konnte damit die Umsetzung der Reorganisationspläne beginnen. Die Stände gaben der Ballei mit dem Beschluss erheblich größere Autonomie, indem sie ihr Befugnisse übertrugen. Jonkheren konnten jetzt ohne aggreatie („Zustimmung“) der Stände zugelassen werden, und die Nachfolge des Landkomturs durch den Koadjutor erfolgte fortan automatisch. Die Stände waren lediglich über das Antreten eines neuen Landkomturs in Kenntnis zu setzen. Das neue Nachfolgeverfahren trat bereits im folgenden Jahr, nach dem Tod Torcks am 5. November 176I, in Kraft. Koadjutor van Wassenaer übernahm direkt dessen Amt. Die Komture und die Stände von Utrecht wurden schriftlich informiert und äußerten ihrerseits schriftlich ihre Zustimmung. Während der Kapitelversammlung von 1762 konnte van Wassenaer die von ihm selbst angestoßene Reorganisation als Landkomtur zum Abschluss bringen. Zu Beginn der Versammlung wurde er im höchsten Amt der Ballei bestätigt. Neuer Koadjutor wurde sein Kommissionskollege van Randwijck. Dieser rückte dem Schema gemäß von der Kommende Maasland zur Kommende Dieren auf, die an das Amt des Koadjutors gekoppelt war. Die Kommenden waren nun leere Titel geworden, die nur den Platz in der Rangordnung wiedergaben und das Recht auf ein bestimmtes Gehalt gewährten.

\section{Die Neuordnung in der Praxis}

Unter der Führung der ehemaligen Kommissionsmitglieder van Wassenaer und van Randwijck und mit Cazius und van Nes in den ausführenden Funktionen konnte die Reorganisation beginnen. Von I. Januar 1762 an wurden die Besitztümer von der Landkommende aus verwaltet, die gleichzeitig Wohnsitz des

22 Brief von U. W. van Wassenaer an F. S. C. van Randwijck, 10.08.1762, GA 609, Inv. Nr. 220, Brieven ingekomen bij F. S. C. van Randwijck als commandeur van Tiel (1754-1763) 1 omslag. 
Generalverwalters war. Von hier aus besuchte dieser die Pächter, um sie zur Zahlung zu mahnen, und um den Zustand der Bauernhöfe und des umliegenden Landes zu beurteilen. Er sorgte für eine ordentliche Buchhaltung, indem er Einnahmen und Ausgaben der gesamten Ballei genau dokumentierte. Endlich wurde ersichtlich, wohin das Geld floss, und wie die Einkünfte erhöht werden konnten. Dies hatte man im finanziellen Chaos, das ein Jahrzehnt zuvor der Anlass für van Wassenaers Notsignal gewesen war, nicht nachvollziehen können. Cazius notierte alles minutiös. In der Generalrechnung stellte er die Einkünfte aus Immobilien den Ausgaben für Komtursgehälter, Steuern und Instandhaltung gegenüber. ${ }^{23} \mathrm{Da}$ neben führte er eine Rechnung für die Ordenskasse. Auf deren Einnahmenseite standen die Aufnahme- und Anmeldegebühren, Überschüsse aus der Generalkasse und der Ordenskasse selbst, ebenso wie ein Teil der Zinsen auf Darlehen und Obligationen. ${ }^{24}$ Der andere Teil letzterer wurde auf der Einnahmenseite der Generalrechnung verbucht. Worauf diese Aufteilung basierte, ist nicht ganz klar. Auch die Ausgaben wurden auf beide Rechnungen verteilt. Das Gehalt des Verwalters belastete die Generalrechnung, jenes des Sekretärs die Rechnung der Kasse. Aufgrund dieses Umstandes ist es für den Historiker schwierig, schnell einen Überblick über die Finanzen der Ballei zu gewinnen. Im Vergleich zur Situation von vor 1762 kann jedoch von einer erheblichen Verbesserung gesprochen werden. Der Generalverwalter besprach die von ihm erstellten Rechnungen mit dem Landkomtur, woraufhin sie vom Kapitel genehmigt werden mussten. Zwischen den Kapitelversammlungen wurden die Rechnungen von einer Kommission kontrolliert. Diese bestand in der Regel aus dem Landkomtur, dem Koadjutor und dem Komtur von Maasland. Sie konnte aber auch anders zusammengesetzt sein. Diese Gruppe, die faktisch als Geschäftsführung auftrat, sollte später als „dirigierende Kommission" bezeichnet werden.

Van Wassenaer wollte die Frequenz der Kapitelversammlungen reduzieren, um Kosten einzusparen. Die Zusammenkünfte der Komture, während derer die Herren im Deutschhaus logierten, waren vor allem aufgrund der Versorgung mit ausgesuchten Speisen und Getränken kostspielige Angelegenheiten. So kostete die Versammlung von 1762, an der sieben Mitglieder teilnahmen, über I 800 Gulden - den siebenfachen Jahreslohn eines Facharbeiters. Die Kosten der Versammlung von 1767 lagen noch 300 Gulden höher. ${ }^{25} \mathrm{Zu}$ diesem Zeitpunkt war van Wassenaer schon nicht mehr im Amt. Er war im November des Vorjahres ver-

23 ARDOU-OA, Inv. Nr. 337, Generale rekeningen van de rentmeesters-generaal van de D.O. van alle commanderijen (1762-1811) 51 Bände.

24 ARDOU-OA, Inv. Nr. 342, Rekeningen van de de rentmeesters-generaal van de D.O. betreffende de orde-kassa (1756-1811) 51 Bände.

25 ARDOU-OA, Inv. Nr. 342, (1767), Fol. 11r. 
storben, und entsprechend der neuen Regeln hatte der damalige Koadjutor van Randwijck seine Nachfolge angetreten. Als Miturheber der Reorganisationspläne führte dieser den eingeschlagenen Kurs mit Entschlossenheit und in gutem Einverständnis mit Verwalter Cazius fort.

Dass die Neuordnung auch finanziell Früchte trug, geht aus den Ergebnissen hervor. Die erste von Cazius präsentierte Generalrechnung, für das Jahr 1762, weist noch ein Defizit von 1396 Gulden auf. Die Ursache ist dabei deutlich: Die Einnahmen beliefen sich auf 28444 Gulden, die Ausgaben auf 29840 Gulden. ${ }^{26}$ Die Generalrechnung für 1763 weist bereits einen Überschuss auf. Wie die nachstehende Tabelle zeigt, sind die Überschüsse in den Folgejahren stetig gewachsen.

Tabelle 1: Einnahmen und Ausgaben der Ballei Utrecht, 1762-1795

\begin{tabular}{|c|c|c|c|}
\hline Jahr & Einnahmen & Ausgaben & Saldo \\
\hline 1762 & 28444 & 29840 & -1396 \\
\hline 1763 & 32246 & 30805 & 1441 \\
\hline 1764 & 30589 & 29562 & 1027 \\
\hline 1765 & 31238 & 30279 & 959 \\
\hline 1766 & 33268 & 30903 & 2365 \\
\hline 1767 & 31951 & 30221 & 1730 \\
\hline 1768 & 32549 & 30954 & 1595 \\
\hline 1769 & 32525 & 30621 & 1904 \\
\hline 1770 & 32566 & 30213 & 2353 \\
\hline 1771 & 32784 & 30950 & 1834 \\
\hline 1772 & 33121 & 30760 & 2361 \\
\hline 1773 & 33684 & 31438 & 2246 \\
\hline 1774 & 35129 & 32251 & 2878 \\
\hline 1775 & 35108 & 31850 & 3258 \\
\hline 1776 & 33317 & 31529 & 1788 \\
\hline 1777 & 35958 & 32618 & 3340 \\
\hline 1778 & 34739 & 32536 & 2203 \\
\hline 1779 & 35661 & 32257 & 3404 \\
\hline 1780 & 37210 & 32106 & 5104 \\
\hline 1781 & 37791 & 32756 & 5035 \\
\hline 1782 & 38039 & 32370 & 5669 \\
\hline 1783 & 37968 & 31577 & 6391 \\
\hline 1784 & 39839 & 36248 & 3591 \\
\hline & & & \\
\hline
\end{tabular}

26 ARDOOU-OA, Inv. Nr. 337. 
Tabelle 1:

\begin{tabular}{|c|c|c|c|}
\hline Jahr & Einnahmen & Ausgaben & Saldo \\
\hline 1785 & 38566 & 36511 & 2055 \\
\hline 1786 & 38979 & 36556 & 2423 \\
\hline 1787 & 39053 & 35640 & 3413 \\
\hline 1788 & 40745 & 37290 & 3455 \\
\hline 1789 & 41296 & 37791 & 3505 \\
\hline 1790 & 41573 & 37190 & 4383 \\
\hline 1791 & 41960 & 36128 & 5832 \\
\hline 1792 & 43504 & 37606 & 5898 \\
\hline 1793 & 44256 & 37939 & 6317 \\
\hline 1794 & 43662 & 38132 & 5530 \\
\hline 1795 & 45117 & 37352 & 7765 \\
\hline
\end{tabular}

Quelle: ARDOU-OA, Inv. Nr. 337, Generalrechnung

Es gelang Cazius schnell, die Einnahmen deutlich zu erhöhen und die Ausgaben gleichzeitig unter Kontrolle zu halten. In einer memorie van voordeelige verrigtingen („Memorandum über vorteilige Verrichtungen“) berichtete er voller Stolz, dass die Einnahmen um 6 a 7000 gulden sjaarlijks gesteijgerd wurden. ${ }^{27}$ Die Hauptursache für die Steigerung waren die verbesserten Pachteinnahmen. Im Jahr 1763 lagen sie bereits fast 3000 Gulden höher als im ersten Jahr des neuen Systems. Die Einnahmensteigerung setzte sich weiter fort, vor allem von der Mitte der I $770 e r$ Jahre an. Im Buchjahr 1763 lag die Gesamtsumme der Pachteinnahmen den Aufzeichnungen Cazius' zufolge bei 20997 Gulden, und im Jahr 1784 bei 29485 Gulden. Damit hatte der Verwalter innerhalb von gut 20 Jahren eine Steigerung von 65 Prozent erzielt.

Und tatsächlich ist sie größtenteils Cazius zu verdanken. In enger Absprache mit dem Landkomtur hatte er die Güterverwaltung verbessert. Unrentable und perifer gelegene Ländereien wurden abgestoßen, sodass nur diejenigen Bauernhöfe übrig blieben, die in Ballungsgebieten und auf fruchtbaren Böden lagen. Dass er die Pachteinnahmen zu erhöhen verstand, hatte Cazius bereits bei der Verwaltung der Güter der Landkommende im Dorf Maasland bewiesen. Diese hatte er im Jahr 1753 in een disperate toestand (,,in desperatem Zustand“) vorgefunden. ${ }^{28}$

27 ARDOU-OA, Inv. Nr. 366, Memorie door de rentmeester-generaal G.D. Cazius ingediend bij de landcommandeur omtrent zijn verrichtingen in het belang van de D.O. op financieel gebied (1763).

28 ARDOU-OA, Inv. Nr. 366. 
Er besuchte die Pächter, um Zahlungsrückstände einzutreiben oder Zahlungsregelungen zu treffen. Diese Vorgehensweise wendete er nach seiner Ernennung zum Generalverwalter auf die gesamte Ballei an. Dass sie schnell Erfolg zeigte, fiel auch dem Landkomtur van Wassenaer auf. Er stellte im August 1763 fest, dass seit der Übernahme der Verwaltung aller Kommenden durch Cazius derselver inkomen werkelijk vermeerdert was („derselben Einkommen sich wirklich vermehrt hatte"), was er vor allem mit Cazius' Administration und persönlichen Besuchen in Verbindung brachte. ${ }^{29}$ Die großen Erfolge standen damals jedoch noch aus. Wie die vorstehende Tabelle zeigt, stiegen die Pachteinnahmen der Ballei vor allem ab Mitte der I $770 e r$ Jahre stark an.

Dieser Erfolg war auch der Wirtschaftslage zu verdanken. In den Jahren nach Cazius' Ernennung stiegen die Korn- und Milchpreise deutlich an. Die zunehmende Nachfrage aus dem sich industrialisierenden England war hierbei ein treibender Faktor. Auch die Kriegszeit, die mit dem Aufstand der amerikanischen Kolonisten I 773 begonnen hatte, wirkte sich preistreibend auf den Nahrungsmittelmarkt aus. Nachteilige Ereignisse wie Viehseuchen und Überschwemmungen, die in den Jahren 1740 und 1750 zu Einbrüchen bei den Pachteinnahmen geführt hatten, traten dagegen immer seltener auf. ${ }^{30}$ Die Bauern konnten ihre Pachten jetzt problemlos zahlen, und Cazius sorgte dafür, dass sie auch tatsächlich eingetrieben wurden. Beim Auslaufen eines Pachtvertrags oder bei der Übernahme durch einen neuen Pächter erhöhte er die Pachtsummen deutlich. Cazius hatte ein gutes Gespür dafür, welche Faktoren die Einnahmen beeinflussten. So bemerkte er zu den Auswirkungen der amerikanischen Revolution: die buijtenlandsche oorlogen sullen soo doende ons landt veel voordeel brengen („die ausländischen Kriege werden unserem Land somit großen Vorteil bringen").31 Auch riet er dem Landkomtur, die Renditen aus dem Landbesitz beispielsweise durch die Umwandlung von Gartenbauland in Weideland zu steigern. Außerdem behielt er stets den Markt im Blick, um gute Böden ankaufen und weniger rentable Ländereien abstoßen zu können.

Die günstige Entwicklung der Pachteinnahmen lässt sich auch bei anderen vergleichbaren Einrichtungen beobachten, etwa bei den ehemaligen Frauenklostern der Provinz Utrecht, die nach der Reformation als Einkommensquelle adliger Damen dienten. Diese konnten die Rendite ihrer Ländereien zwischen 1760 und I 790 um den Faktor I, 33 steigern. Die Ballei Utrecht erreichte gar den Faktor

\footnotetext{
29 Brief von U. W. van Wassenaer an die Mitglieder des Kapitels, 27.08.1763, GA 609, Inv. Nr. 224.

30 Rommes (wie Anm. 16); de Vries, van der Woude (wie Anm. 7) S. 270-274.

31 Brief von G. D. Cazius an F. S. C. van Randwijck, 19.09.1778, GA 609, Inv. Nr. 222.
} 
1,46. ${ }^{32}$ Auch die Privateigentümer konnten im selben Zeitraum die Einnahmen aus ihrem Landbesitz steigern. Nicht zuletzt aus diesem Grund gelang es dem niederländischen Adel, seine im Zuge der Landwirtschaftskrise geschwächte Position in der zweiten Hälfte des I 8. Jahrhunderts wieder zu festigen. ${ }^{33}$

Was die Einkommensseite betraf, wurde Cazius durch die Konjunktur unterstützt, doch die Kontrolle der Ausgaben war zweifellos sein eigener Verdienst. Die Steigerung der Ausgaben blieb deutlich hinter derjenigen der Einnahmen zurück, sodass die Überschüsse stetig wuchsen. Den größten Kostenposten stellten die Gehälter der Kapitelmitglieder dar. Sie beliefen sich zunächst auf I 4300 Gulden, und nach der Erhöhung im Jahr I 783 auf 17400 Gulden pro Jahr. Die wechselnden Beträge für die Instandhaltung von Bauernhöfen und Gebäuden lagen im Schnitt bei 2500 Gulden pro Jahr. Die teuerste Immobilie war das Deutschhaus in Utrecht, an dem regelmäßig Zimmerleute, Maurer, Dachdecker und Maler Arbeiten verrichteten. Mit diesen führte Cazius harte Verhandlungen. Er wusste, dass er sich dabei keine Freunde machte: de luijden werden boos tegen mij, als ik niet wil toelaaten dat de order banaelt werd („die Leute werden böse auf mich, wenn ich nicht zulassen will, dass der Orden benachteiligt wird“), schrieb er an Landkomtur van Randwijck. ${ }^{34}$ Auch den Mitgliedern des Kapitels gegenüber setzte er seinen Sparkurs durch. Im Jahr 1767 weigerte er sich, dem Sohn des neuen Koadjutors Frederik Hendrik van Wassenaer van Catwijk ( I 70 I-I 77 I) eine Geldsumme auszuzahlen - zum großen Ärger von dessen Gattin.

Sehr wenig Geld wurde für kirchliche und soziale Zwecke ausgegeben. Im Jahr I 763 waren auf der Generalrechnung 452 Gulden (I,5\%) an kirchlichen Posten vermerkt, darunter ein Beitrag zu einem Pastorengehalt und ein Beitrag von 179 Gulden ( $0,6 \%$ ) für die Armenfürsorge. ${ }^{35}$ Darüber hinaus gab es vereinzelte Schenkungen von einer Dukate, zwei Gulden, einer Flasche Wein und zwei Kissen für einen Handwerker, der bei Arbeiten am Deutschhaus vom Dach gefallen war und sich das Bein gebrochen hatte. Von einer karitativen Einrichtung konnte bei der Ballei Utrecht nicht die Rede sein. Van Wassenaer hatte in seinem Reformplan die Zuwendungen für die Komture und die gute Instandhaltung der Besitztü-

32 W. van den Brink, Utrechtse adellijke vrouwen en prebenden in de achttiende eeuw, Virtus. Jaarboek voor Adelsgeschiedenis 11 (2004) S. 47.

33 P. G. Brusse, W. W. Mijnhardt, Towards a new template for Dutch History, Virtus. Yearbook of the History of the Nobility 19 (2012), S. 173-180; R. E. de Bruin, Utrechter Patrizier und Bürger als Gutsbesitzer. Ein Aristokratisierungsprozess, 1600-1850, in: Städtische Siedlungen und ihr Umland. Siedlungsforschung, Archäologie - Geschichte - Geographie 26, hrsg. v. J. Renes, W. Schenk, Bonn 2008, S. 87-126.

34 Brief van G. D. Cazius aan F. S. C. van Randwijck, 10.11.1767, GA 609, Inv. Nr. 222.

35 ARDOU-OU, Inv. Nr. 337, Band 2 (1763). 
mer in den Vordergrund gestellt. Von den einstigen Idealen, dem Kampf für das Kreuz und der Versorgung von Kranken und Verwundeten, war nicht viel übrig geblieben: Der Orden kümmerte sich vorwiegend um den Lebensunterhalt für Brüder („Spital des deutschen Adels"), und innerhalb der Ballei Utrecht dienten die Ordensämter nur noch als lukrativer Nebenverdienst und zur Steigerung des Prestiges verheirateter Adliger. Letzteres ergab sich vor allem aus der Exklusivität der Mitgliedschaft, die sich in der Forderung nach vier adligen Großelternteilen äußerte. In der niederländischen Republik erfuhr der Adel zunehmende Konkurrenz durch Patrizier und Kaufleute, die aristokratische Ambitionen an den Tag legten. Da es in der Republik anders als in den sie umgebenden Monarchien keine Nobilitierung gab, kauften diese Bürger Schlösser und herrschaftliche Titel, um sich einen adligen Lebensstil anzueignen. Der alte Adel wehrte sich hiergegen und erreichte immerhin, dass die Ritterschaften für Neulinge unzugänglich blieben. Während der Landwirtschaftskrise von 1650-1750 waren die Söhne mehrerer Adelsgeschlechter gezwungen, bürgerliche Mädchen zu ehelichen, um finanziell den Kopf über Wasser zu halten. Eine solche Ehe disqualifizierte die aus ihr hervorgegangenen Nachkommen für die Mitgliedschaft im Deutschen Orden. Wer dagegen die Voraussetzungen erfüllte, konnte durch seine Mitgliedschaft seine rein adlige Abstammung zur Schau tragen. ${ }^{36}$

\section{Die Mitglieder der Ballei Utrecht}

Bei der Zulassung von Mitgliedern und der Verteilung der Aufgaben unter ebendiesen hatte die Ballei Utrecht in den Jahren $1760-1762$ ein großes Maß an Autonomie erworben. Wie schon erwähnt, verlief die Nachfolge des Landkomturs durch den Koadjutor automatisch: 176 I folgte van Wassenaer auf Torck und fünf Jahre später van Randwijck auf van Wassenaer. Van Randwijck blieb fast 20 Jahre im Amt, sein Nachfolger sogar etwas über 20 Jahre. Der Landkomtur hatte jetzt viel mehr Macht als vor dem Beginn der Neuordnung im Jahr 1753. Torck, van Wassenaer und van Randwijck nahmen ihre Aufgabe auch viel ernster als ihre Vorgänger. Mit Ausnahme von Godard van Reede van Athlone hatten diese nicht besonders viel Interesse an der Institution gezeigt. Van Wassenaer und van Randwijck unterhielten eine rege Korrespondenz mit dem Generalverwalter und hatten auch untereinander regelmäßig Kontakt. Und obwohl diese beiden Landkomture

36 R. E. de Bruin, De Ridderlijke Duitse Orde Balije van Utrecht, een adellijk distinctie-instrument in de Republiek der Verenigde Nederlanden gedurende de achttiende eeuw, Virtus. Jaarboek voor Adelsgeschiedenis 16 (2009) S. 34-53. 
keinen Gebrauch von ihrem Wohnrecht machten, waren sie doch regelmäßig in der Landkommende zugegen. Zwischen den Kapitelversammlungen kontrollierte der Landkomtur zusammen mit zwei weiteren Kommissionsmitgliedern die Rechnungen des Generalverwalters, und er traf Entscheidungen, die nicht bis zur nächsten Vollversammlung warten konnten.

Auch in der Aufnahme von Mitgliedern konnte die Ballei Utrecht jetzt ihren eigenen Weg gehen. Wenn ein Kapitelmitglied verstarb, so rückten alle Komture um einen Rang auf, und der erste jonkheer wurde zum Komtur des untersten Ranges ernannt. Anschließend erhielt der erste Expektant auf der Liste die Aufforderung, die Nachweise über seine adlige Abstammung und das reformierte Glaubensbekenntnis beizubringen. Die Kontrolle oblag zwar dem Kapitel selbst, doch galten dabei die strengen Regeln aus der Satzung von 1740, die 1755 bestätigt und 1756 durch die Stände von Utrecht genehmigt worden war. Dass die Kontrolle ernst genommen wurde, zeigt sich auch daran, dass mehrere Mitgliedschaftsanfragen abgelehnt wurden. Gründe waren beispielsweise die falsche Variante des Protestantismus (lutherisch), die Konversion zum Katholizismus, aber auch eine ungeeignete Abstammung. Ironischerweise wurde auch der Sohn Unico van Wassenaers nicht zugelassen. Grund war seine friesische Abstammung mütterlicherseits. Van Wassenaer Sr. war mit einer Tochter einer einflussreichen friesischen Familie verheiratet. Da die Provinz Friesland keine Ritterschaft kannte, war der Status des friesischen Adels problematisch. ${ }^{37}$

Prinzipiell kamen nur Angehörige niederländischer rittermäßiger Geschlechter als Mitglieder der Ballei Utrecht in Frage, doch angesichts der traditionellen Bande durften auch deutsche Adlige beitreten. Sie mussten dann genau wie die Niederländer den Nachweis über die Abstammung von vier adligen Großelternteilen und die Zugehörigkeit zu einer reformierten Glaubensgemeinschaft erbringen. Letztere war in den deutschen Ländern viel weniger selbstverständlich als in der niederländischen Republik, doch gab es auch im Deutschen Reich unter den adligen und fürstlichen Familien relativ viele Calvinisten. Das Hindernis der abweichenden Religion (lutherisch oder katholisch) trat ausschließlich bei deutschen Kandidaten auf. Die Kontrolle der Nachweise war auch ein sprachliches Problem: In der Ballei Utrecht konnte man kein Deutsch lesen, was wahrscheinlich vor allem an der gotischen Schrift lag. Deutsche Dokumente wurden durch einen in Utrecht wohnhaften Deutschen, den „Translateur" Johann Casimir Knackwoerst ( $\dagger_{\text {I }} 805$ ), übersetzt. Um innerhalb der Ballei funktionieren zu können, bedienten sich die deutschen Mitglieder des Französischen, oder sie lernten

37 Y. Kuiper, Adel in Friesland 1780-1880, Groningen 1990, S. 82-84; J. Streng, De adel in de Republiek, Virtus. Jaarboek voor Adelsgeschiedenis 10 (2003), S. 73-74. 
Niederländisch. Letzteres war nicht so schwierig, da die deutschen Mitglieder als Offiziere in der niederländischen Armee gedient hatten, oder aber aus dem Herzogtum Kleve stammten, das enge Bande mit der benachbarten niederländischen Provinz Gelderland unterhielt, und dessen Umgangssprache noch eng mit dem Niederländischen verwandt war. ${ }^{38}$ Im Zeitraum $1753-1795$ hat es sechs Komture mit deutschem Hintergrund gegeben. Einer von ihnen, Karl Ludwig von AnhaltBernburg-Schaumburg-Hoym (1723-I 806), brachte es sogar zum Landkomtur. Er hatte als junger Fürstensohn in der niederländischen Armee gedient und war I 767 dem Kapitel der Ballei Utrecht beigetreten. Einige Jahre später war er in sein Geburtsland zurückgekehrt, um die Nachfolge seines verstorbenen Vaters anzutreten.

Die niederländischen Mitglieder der Ballei stammten größtenteils aus den östlichen Provinzen Gelderland und Overijssel. Einige kamen aus Utrecht oder aus Holland. Die Provinzen Seeland, Friesland und Groningen waren im Kapitel nicht vertreten; sie kannten keine Ritterschaft. Die meisten Komture waren über die Ritterschaften Mitglied der Ständeversammlung ihrer Provinz und erfüllten von hier aus auch Ämter in den Generalständen oder im diplomatischen Dienst der Republik. Wer nicht Mitglied der Stände war, war Offizier in der Armee, wie etwa der Landkomtur van Randwijck, der seine militärische Laufbahn als Generalleutnant beendete.

Durch ihre starke Verflechtung mit der niederländischen Politik waren die Komture der Ballei Utrecht auch regelmäßig an den Konflikten beteiligt, die die Republik zerrissen, und die sich im Kampf zwischen den Anhängern des Prinzen von Oranien (de prinsgezinden) und dessen Gegnern (de staatsgezinden) zuspitzten. ${ }^{39}$ Die prinsgezinden wollten in der föderativen Republik der Vereinigten Niederlande die zentralistischen und monarchischen Elemente stärken, und forderten mehr Macht für die Statthalter aus dem Hause Nassau, also die Nachkommen der Anführer des Aufstands gegen die Spanier, Wilhelm von Oranien (1533-1584) und Johann VI. Graf von Nassau-Dillenburg (1536-1606). Die staatsgezinden wollten deren Macht im Gegenteil beschneiden. Sie erlebten ihre größten Triumphe in den Zeiträumen 1650-1672 und 1702-1747, den „Statthalterlosen Perioden“, in denen es in den meisten Provinzen, auch in der mächtigen Provinz Holland, keinen Statthalter gab. Diese Situation bezeichneten sie als „wahre Freiheit", als Verwirklichung des republikanischen Ideals der weitgehenden

38 N. van der Sijs, De geschiedenis van de Nederlandse taal, in: Dialectatlas van het Nederlands, hrsg. v. N. van der Sijs, Amsterdam 2011, S. 74-75.

39 H. Lademacher, Geschichte der Niederlande. Politik, Verfassung, Wirtschaft, Darmstadt 1983, S. 100-126, 161-172; Israel (wie Anm. 8) S. 687-704, 799-980, 1089-1097. 
Autonomie von Provinzen und Städten. ${ }^{40}$ Der Gegensatz zwischen beiden Lagern hatte auch internationale Implikationen: Die prinsgezinden wünschten in der AuBenpolitik einen pro-englischen Kurs, die staatsgezinden strebten nach Neutralität oder hegten leichte Sympathien für Frankreich. Mit der Installation Wilhelms IV. als allgemeinem Erbstatthalter (infolge des französischen Einfalls) im Jahr 1747 brach eine Zeit an, in der die niederländische Republik einen semi-monarchalen Charakter bekam. Dies wurde offensichtlich, als nach dem plötzlichen Tod des Statthalters im Jahr I75 I dessen dreijähriger Sohn Wilhelm V. (I748- I 806) zu seinem Nachfolger wurde. Seine Mutter Anna von Hannover ( I 709-1 759) trat als Regentin auf. Sie war die Tochter von König George II. Hieran zeigt sich, wie eng die statthalterliche Familie mit Großbritannien verbunden war.

Im 17. Jahrhundert hatte die Ballei Utrecht auf der Linie der Oranien-Anhänger gelegen: Drei Landkomture entstammten dem Hause Nassau, und nach ihnen bekleidete ein Vertrauter Wilhelms III. (Godard van Reede van Athlone) das Amt. Mitte des folgenden Jahrhunderts war die Situation verändert: Die Landkomture Hendrik van Isselmuden, Frederik Willem Torck und Unico Wilhelm van Wassenaer waren erklärte staatsgezinden, die den politischen Umschwung von 1747 mit Sorge betrachteten. Van Wassenaer bezog außenpolitisch einen ausgesprochen anti-britischen Standpunkt. Seine diplomatischen Aktivitäten während des Österreichischen Erbfolgekriegs zeugten von guten Kontakten nach Frankreich und Köln. Beim folgenden internationalen Konflikt, dem Siebenjährigen Krieg, plädierte er erfolgreich für Neutralität anstelle der Unterstützung Großbritanniens - zur großen Verärgerung von Prinzessin Anna. ${ }^{41}$

Nach dem Tod van Wassenaers im Jahr 1766 sympathisierte das Kapitel immer mehr mit dem Statthalter. Im Oktober 1770 wurde deutlich, wie eng die Beziehungen inzwischen geworden waren: Die Kapitelversammlung beschloss, das ungeborene Kind Prinzessin Wilhelmines von Preußen, Nichte Friedrichs des Großen und Ehefrau des Statthalters Wilhelm V., als Expektant der Ballei vorzutragen. Da sich herausstellte, dass es sich um ein Mädchen (Luise) handelte, kam es nicht zur Anmeldung. Als die Ritter dies auf der folgenden Kapitelversammlung enttäuscht feststellten, hatte die Prinzessin jedoch bereits einen Sohn geboren (den späteren König Wilhelm I.), der direkt angemeldet werden konnte. ${ }^{42}$ Einige Jahre später folgte dessen Bruder Friedrich.

Nun war es im statthalterlichen System kaum möglich, Opposition zu machen, doch auch als in den $1780 e r$ Jahren neue politische Spannungen entstanden und gar

40 Lademacher (wie Anm. 39), S. 114-126, 167-172.

41 N. A. Bootsma, De Hertog van Brunswijk 1750-1759, Assen 1962, S. 276-277.

42 ARDOU-OU, Inv. Nr. 11-3, Fol. 157 v. 
eine revolutionäre Stimmung aufkam, blieben die meisten Komture Wilhelm V. treu. Einige von ihnen, wie Joost Taets van Amerongen (1726-I79I), Johan Walraat Graf von Welderen (1725-1 807) und Sigismund Vincent Lodewijk van Heiden-Hompesch (173I-1790) waren in ihrer jeweiligen Provinz - Utrecht, Gelderland und Overijssel - sogar wichtige Unterstützer im Kampf gegen die nahende Revolution. Zwei Expektanten, die einige Jahre später beitreten sollten, beteiligten sich im Sommer 1787 an Prinzessin Wilhelmines Versuch, die Revolution mit britischer Unterstützung zu unterdrücken. Dieser missglückte zwar, doch kam es in seiner Folge zur Intervention durch Wilhelmines Bruder, König Friedrich Wilhelm II. von Preußen. Dieser setzte der Revolution im September 1787 ein Ende.

\section{VersuChe DeR Wiedervereinigung Mit DEM Hochmeister}

Die durch die Reorganisation erlangte größere Autonomie eröffnete auch die Möglichkeit zum Dialog mit dem Hochmeister. Nach dem Bruch im Jahr I 640 war der Sitz Utrechts im Kapitelsaal in Mergentheim unbesetzt geblieben. ${ }^{43}$ Seitens des Hochmeisters war es stets die Intention gewesen, den Bruch einst zu heilen. Über die Ballei Biesen waren in den Jahren I 667, I672 und I685 Versuche unternommen worden, die Ballei Utrecht wieder in die Ordensgemeinschaft einzugliedern. Seitens der Ballei, unter anderem bei Landkomtur Floris Borre van Amerongen (1602-1675), bestand auch durchaus Bereitschaft hierzu, doch die Stände von Utrecht blockierten in ihrer Eigenschaft als Souverän der Ballei jegliche Annäherung. ${ }^{44}$

Nachdem die Stände im Rahmen der Neuordnung allerdings auf Kontrollbefugnisse gegenüber der Ballei verzichtet hatten, schien es möglich, dass sie auch ihre Blockadehaltung aufgeben könnten. Aus dieser Erwägung heraus wurde I 3 Jahre nach Inkrafttreten der Neuordnung ein neuer Vorschlag zur Wiedervereinigung gemacht. Die Initiative dazu kam von Hochmeister Karl Alexander von Lothringen ( $1712-1780$ ), dem jüngeren Bruder Kaiser Franz' I., der für seine Schwägerin Maria Theresia als Generalgouverneur die Österreichischen Niederlande verwaltete. Er hatte 176I die Nachfolge des Hochmeisters Clemens August von Wittelsbach angetreten und sich zum Ziel gesetzt, den Niedergang des

43 Sitzordnung des Generalkapitels Mergentheim, 1791 (?), Deutschordens-Zentralarchiv, Wien, GK 754/2, S. 631 f.; Arnold (wie Anm. 7), S. 256.

44 De Vey Mestdagh (wie Anm. 7) S. 48-53; De Bruin, Bedreigd door Napoleon (wie Anm. 2) S. 66-67. 
Ordens durch eine Änderung der Statuten von 1606 und die Rückgewinnung verloren gegangener Teile aufzuhalten und umzukehren. Er gab hierzu Recherchen in Auftrag, im Rahmen derer auch die missglückten Wiedervereinigungsversuche mit Utrecht ein Jahrhundert zuvor auf den Tisch kamen. Sie inspirierten ihn zu einem neuen Versuch, der ebenfalls über die Ballei Biesen lief. Im Frühjahr I 775 führte der Hochmeister mit dem Biesener Landkomtur, Caspar Anton von der Heyden genannt Belderbusch (1722-1784), ein Gespräch in Sachen Utrecht. ${ }^{45}$ Der Vertraute des Hochmeisters erörterte die Lage. Im Februar 1776 eröffnete der Hochmeister die Gespräche mit Utrecht. Ein weiterer Vertrauter, der Landkomtur der Ballei Elsass-Burgund, Beat Conrad Philipp Friedrich Reuttner von Weyl (1719-1803), nahm mit dem Utrechter Kapitelmitglied Graf von Welderen Kontakt auf. Sie besprachen einen in Mergentheim aufgestellten Elfpunkteplan, der die Anerkennung der Oberhoheit des Hochmeisters und damit die Ernennung der Utrechter Kapitelmitglieder durch den Hochmeister, die Leistung einer jährlichen Abgabe und die Präsenz des Utrechter Landkomturs bei den Kapitelversammlungen in Mergentheim beinhaltete. Die Ballei Utrecht sollte im Gegenzug ihre reformierte Ausrichtung behalten dürfen, und die Mitglieder sollten von der Zölibatsverpflichtung, dem Noviziat in Mergentheim und dem Militärdienst für den Orden freigestellt sein. ${ }^{46}$

Van Welderen sandte den in französischer Sprache verfassten Vorschlag an seine Kapitelkollegen. Diese hatten so einige Fragen hinsichtlich der souveränen Kontrolle der Utrechter Stände über die Ballei, der Zölibatsfrage und der Abgabe an den Hochmeister. Reuttner von Weyl gab sich in seiner Reaktion größte Mühe, die Utrechter Ritter zu beschwichtigen. Er garantierte, dass Beschlüsse zur Wiedervereinigung nicht contraires à la constitution de la République et à l'usage jusquien adopté $d u$ Baillage sein würden. Was die Keuschheitsforderung betraf, erklärte er, dass une vie chretienne et chaste même dans le mariage ausreichend sei. Hinsichtlich der Abgaben an Mergentheim versprach er, dass sie de la plus grande modération sein würden. ${ }^{47}$

45 B. Demel, Die Deutschordensballei Utrecht in der Reichs- und Ordensüberlieferung von der frühen Neuzeit bis in die Zeit Napoleons, in: Unbekannte Aspekte der Geschichte des Deutschen Ordens, hrsg. v. B. Demel, Wien-Köln-Weimar, 2005, S. 83.

46 ARDOU-OA, Inv. Nr. 131, Briefwisseling gevoerd tussen de administrateur van de hoogmeester, de landcommandeur van Alden Biesen en de stadhouder van de Balije van Lotharingen met de landcommandeur van Utrecht over de hereniging van de Balije van Utrecht met de D.O. (1662-1672, 1775, 1791-1805) 1 pak, 51: Projet d'une réunion du Baillage d'Utrecht avec les autres Baillages de l'Ordre Teutonique concerté en 1775 entre Mr. le Comte de Welderen et Mr. Le Baron de Reuttner de Weijl, Stadhalter du Baillage d'Alsace.

47 ARDOU-OA, Inv. Nr. 131. 
Doch die Bedenken konnte er hierdurch nicht ausräumen. Vor allem der Komtur von Tiel, Jacob Henrik Graaf van Rechteren (1709-1783), wehrte sich heftig gegen den Vorschlag. Er hielt Rücksprache mit Baron von Stein, einem Komtur der Ballei Hessen. Diese Ballei stand der Ballei Utrecht am nächsten, da sie teilweise calvinistisch war. Außerdem besprach er die Angelegenheit mit Statthalter Wilhelm V., der Einwände gegen die Wiedervereinigung hatte. Mit derartiger Rückendeckung ausgestattet, verfasste van Rechteren einen Bericht, in dem er seine Argumente darlegte. Er fürchtete, dass eine Anerkennung der Oberhoheit des Hochmeisters - unter anderem aufgrund der hierdurch erforderlich werdenden Reisen nach Mergentheim - hohe Kosten mit sich bringen würde, und dass Konflikte mit den Utrechter Ständen entstehen würden. Auch wies er auf Risiken hinsichtlich des persönlichen Erbrechts der Komture hin. Die Einwände waren überzeugend. Der Wiedervereinigungsvorschlag wurde vom Utrechter Kapitel nicht behandelt.

Fünfzehn Jahre später, im Jahr I79 I, lag erneut ein konkreter Vorschlag zur Wiedervereinigung mit dem Hochmeister auf dem Tisch. Inzwischen war die Stimmung günstiger. Van Welderen war zum Koadjutor aufgestiegen, und die Ballei Utrecht hatte mit Fürst Karl Ludwig von Anhalt-Bernburg-Schaumburg-Hoym einen deutschen Landkomtur. Angesichts von dessen Hintergrund lag ein Anschluss an die Balleien des Heiligen Römischen Reiches auf der Hand. Bereits auf der ersten Kapitelversammlung unter seinem Vorsitz, im September 1 786, brachte er vor, dass es tot meerder luijster voor deze Balije zoude strekken („dieser Ballei zu mehr Glanz verhelfen würde"), wenn ihre Komture genau wie die commandeuren van de Balijen der Duitsche Orden in Duitsland eine Uniform trügen, und zwar eine ebensolche..$^{48}$ Der Landkomtur konnte das Kapitel überzeugen, und die Ballei Biesen sollte um ein Modell gebeten werden. Van Welderen erhielt den Auftrag, sich hier um zu kümmern. Er nutzte den Auftrag, um sein Streben nach Wiedervereinigung wieder aufzugreifen. Ein Gespräch mit dem Syndicus von Biesen, Konrad Joseph Bachem ( $\dagger_{\text {I }} 824$ ), brachte geeignete Anknüpfungspunkte. Bachum war Hofrat und Vertrauter des Hochmeisters Maximilian Franz von Österreich (1756-1 80I), der 1780 die Nachfolge Karl Alexanders von Lothringen angetreten hatte. Genau wie sein Vorgänger war Maximilian Franz, ein Bruder Josephs II., für Reformen offen und in religiösen Fragen tolerant. Somit waren auch Konzessionen an die reformierte niederländische Ballei denkbar. Als Modell diente die trikonfessionelle Ballei Hessen. Neue Mitglieder sollten durch einen katholischen Priester installiert werden, jedoch zonder praejudicie van de gereformeerde religie

\footnotetext{
48 ARDOU-OA, Inv. Nr. 11-3, Fol. 214r.
} 
(„ohne Benachteiligung der reformierten Religion“).49 Der Knackpunkt war die auf Neumitglieder bezogene Zölibatsforderung. Sie erntete während der Behandlung des Vorschlags Kritik. Das Kapitel beschloss, als unverhandelbare Bedingung für eine Wiedervereinigung zu fordern que les commandeurs du Baillage d'Utrecht ayent la permission de se marier et de tester [...] selon les uses et coutumes de ces provinces..$^{\circ}$ Van Welderen und der Komtur von Schoonhoven, Jacob Derk Carel van Heeckeren van Kell ( 1730-1795), erhielten den Auftrag, die Reaktion der Utrechter auf den Wiedervereinigungsvorschlag mit den Gesandten des Hochmeisters zu besprechen. Das Verhandlungsergebnis sollte dem Kapitel auf der folgenden Versammlung vorgelegt werden. Dazu ist es jedoch nicht mehr gekommen. Zum Zeitpunkt der nächsten Kapitelversammlung im Jahr I 802 hatte sich die Situation des Deutschen Ordens im Reich und in den Niederlanden dramatisch verändert. Die französischen revolutionären Armeen hatten eine neue Realität geschaffen, in der die Erben der geistlichen Ritterorden aus der Zeit der Kreuzzüge ernsthaft bedroht wurden. Der Kampf, der weniger als ein Jahr nach der Kapitelversammlung I79 I ausgebrochen war, hatte alle Aufmerksamkeit in Anspruch genommen. Einen weiteren Wiedervereinigungsversuch gab es im Jahr I 803. Es handelte sich dabei jedoch um eine rein persönliche Initiative des Komturs Jan Arend de Vos van Steenwijk (1746- I 8 I 3), die im Prinzip von Anfang an zum Scheitern verurteilt war. Der Vorschlag aus dem Jahr I 79 I war die realistischste Chance gewesen, den Bruch zwischen Utrecht und dem Hochmeister zu heilen.

\section{Die Neuordnung Und DER Zeitgeist}

Die durch Unico Wilhelm van Wassenaer initiierte Neuordnung der Ballei Utrecht hat deren Niedergang gestoppt und sie wieder zu einer gut funktionierenden Einheit gemacht, die ihren Mitgliedern große finanzielle Vorteile bescherte. In ihrer Rationalität und Zielstrebigkeit scheinen die Reformvorschläge den Geist der Aufklärung zu atmen. Das für die Aufklärung kennzeichnende utilitaristische Denken tritt aus jeder Argumentation hervor: Die Ländereien seien optimal zu nutzen, Gebäude gut instand zu halten oder abzustoßen, die Finanzen zentral und ordentlich zu verwalten und das Personal nach Eignung auszuwählen. Derartige Leitlinien kehren auch in den Ausführungen der aufgeklärten Denker jener Zeit stets wieder. Die strengere Kontrolle der adligen Abstammung und des reformierten Glaubensbekenntnisses bei der Zulassung neuer Mitglieder dagegen steht in

49 ARDOU-OA, Inv. Nr. 11-4, Fol. 14r.

50 ARDOU-OA, Inv. Nr. 11-4, Fol. 16r. 
krassem Widerspruch zum Gleichheits- und Toleranzideal der Aufklärung. Nun war die niederländische Aufklärung viel weniger radikal als die egalitäre und antiklerikale französische. Sie hatte im Gegenteil sogar einen christlichen Charakter. Dieser basierte allerdings auf einem toleranten und undogmatischen Christentum. ${ }^{51}$ Außerdem förderte die niederländische Aufklärung die Emanzipation des Bürgertums gegenüber Adel und Patriziat. Auch die historischen Argumente van Wassenaers in seinen Plädoyers für die Reformagenda scheinen mit der Aufklärung und deren Zukunftsorientierung und Fortschrittsglauben im Widerspruch zu stehen. Eine ähnliche Argumentationsweise lässt sich allerdings auch bei den politischen Denkern der niederländischen Aufklärung beobachten, und dies sogar in der revolutionären Patriottentijd („Patriotenzeit“). . $^{2}$

Das Nebeneinander von Modernität und Traditionalismus, das die gesamte Neuordnungsphase kennzeichnet, ist zum großen Teil der Person van Wassenaers zuzuschreiben. Er hatte einerseits großes Interesse an der Vergangenheit, wie seine private Ahnenforschung belegt, ging andererseits aber auch mit der Zeit, was sich etwa daran zeigt, dass er die Gärten von Schloss Twickel durch den gefragten Architekten Daniel Marot (1663-1752) umgestalten ließ.93 Die rationale und utilitaristische Herangehensweise, die sich bei der Neuordnung der Ballei Utrecht beobachten lässt, wendete van Wassenaer auch bei der Leitung seiner Landgüter an. Er beschäftigte sich intensiv mit Pachtverträgen und der Instruktion des Verwalters, der genau wie Cazius bei der Ballei Utrecht eine Erhöhung der Pachtabgaben umsetzen sollte. Ein nicht funktionierender Schulmeister wurde von ihm ohne Pardon entlassen. Dass er sich in das Leben seiner Pächter einmischte, indem er übermäßigen Alkoholkonsum zu verhindern suchte, lässt sich noch mit dem Tugendgedanken der Aufklärung in Einklang bringen. Sein Verbot von Mischehen dagegen hat mit der Aufklärung nichts zu tun und lässt sich vielmehr auf seine streng calvinistische Lebensanschauung zurückführen. Diese war auch auf seiner diplomatischen Mission in Köln zutage getreten: Hier störte er sich an der domme religiedrift („tumben Religiosität“) am kurfürstlichen Hof und an „der Bigotterie“ des Kurfürsten und Hochmeisters Clemens August selbst. ${ }^{54}$ Er war froh, dass er

51 W. W Mijnhardt, The Dutch Enlightenment (1650-1800): problems and definitions, in: Centres et péripheries: Les Lumières de Belfast à Bejing, hrsg. v. Marie-Chri Skuncke Paris 2003, S. 170-183; W. W. Mijnhardt, Urbanization, Culture and the Dutch Origins of the European Enlightenment, BMGN The Low Countries Historical Review 125 (2010) S. 141-178.

52 I. L. Leeb, The Ideological Origins of the Batavian Revolution. History and Politics in the Dutch Republic 1747-1800, The Hague 1973.

53 Van der Klooster (wie Anm. 12) S. 101-103.

54 J. Aalbers, 'Rond een bezending naar Munsterland', in: Unico Wilhelm van Wassenaer 1692-1766. Componist en staatsman, hrsg. v. R. Rasch, K. Vlaardingerbroek, Zutphen 1993, S. 206; K. Pörnbacher, 'Daß die Untertanen nach aller Gerechtigkeit geführt und regiert werden. Crescen- 
das Osterfest im Jahr 1745 in einer „ordentlichen“ reformierten Kirche im Herzogtum Berg feiern konnte. Die Verschärfung der Abstammungsbedingungen dagegen ging nicht mit seiner persönlichen Lebensweise zusammen. Er war mit einer Friesin verheiratet, und wie schon erwähnt waren die Probleme mit der Anerkennung friesischen Adels später der Grund, warum seinem Sohn Carel George die Mitgliedschaft im Kapitel verweigert wurde. Die strengeren Anforderungen an die Abstammung gingen auf die Forderungen der Stände von Utrecht zurück.

Die 1753 begonnene Neuordnung beruhte offensichtlich vor allem auf pragmatischen Erwägungen. Van Wassenaer analysierte den Zustand der Ballei genau und schlug zur Lösung der Probleme konkrete Maßnahmen vor. Dabei bediente er sich in großem Maße seiner eigenen Erfahrungen als Besitzer ausgedehnter Landgüter. Sowohl privat als auch in seiner Funktion bei der Ballei Utrecht erwies er sich als fähiger Organisator mit gutem Gespür für Prioritäten. Für das Gelingen der Neuordnung war es äußerst hilfreich, dass sein Nachfolger van Randwijck seine Sichtweise teilte. Auch dieser hatte seine Verbundenheit mit dem Orden unter Beweis gestellt und erkannt, wie wichtig eine gute Verwaltung war. Da van Randwijck 20 Jahre im Amt blieb, stand auch ausreichend Zeit zur Durchführung der Reorganisation zur Verfügung. Auch die hervorragende Zusammenarbeit zwischen van Randwijck und dem von seinem Vorgänger angestellten, äußerst kompetenten Verwalter Gijsberg Dirk Cazius war ein begünstigender Faktor. Die Entscheidungen und Fähigkeiten von Einzelpersonen haben für den Erfolg der Neuordnung eine wichtige Rolle gespielt. Allerdings profitierten diese Akteure auch stark von der wirtschaftlichen und politischen Lage. Cazius wusste die Pachteinnahmen deutlich zu erhöhen, indem er Zahlungen eintrieb und gute Verträge abschloss. Möglich war dies jedoch nur, weil sich die Landwirtschaft im Aufwind befand und die Bauern dank stetig steigender Nahrungsmittelpreise gut bei Kasse waren. Vierzig Jahre nachdem van Wassenaer den lamentablen Zustand der Ballei Utrecht thematisiert hatte, stand dieser isolierte Zweig des Deutschen Ordens wieder hervorragend da. Doch es drohte bereits neue Gefahr: Die Französische Revolution, die sowohl die Balleien im Deutschen Reich als auch die Ballei Utrecht hart treffen sollte.

tia Höß von Kaufbeuren als geistliche Beraterin des Kurfürsten Clemens August', in: Der Riss im Himmel. Clemens August und seine Epoche, Bd. 5: Hirt und Herde. Religiosität und Frömmigkeit im Rheinland des 18. Jahrbunderts, hrsg. v. F. G. Zehnder, W. Schäfke, Köln 2000, S. 197-212. 


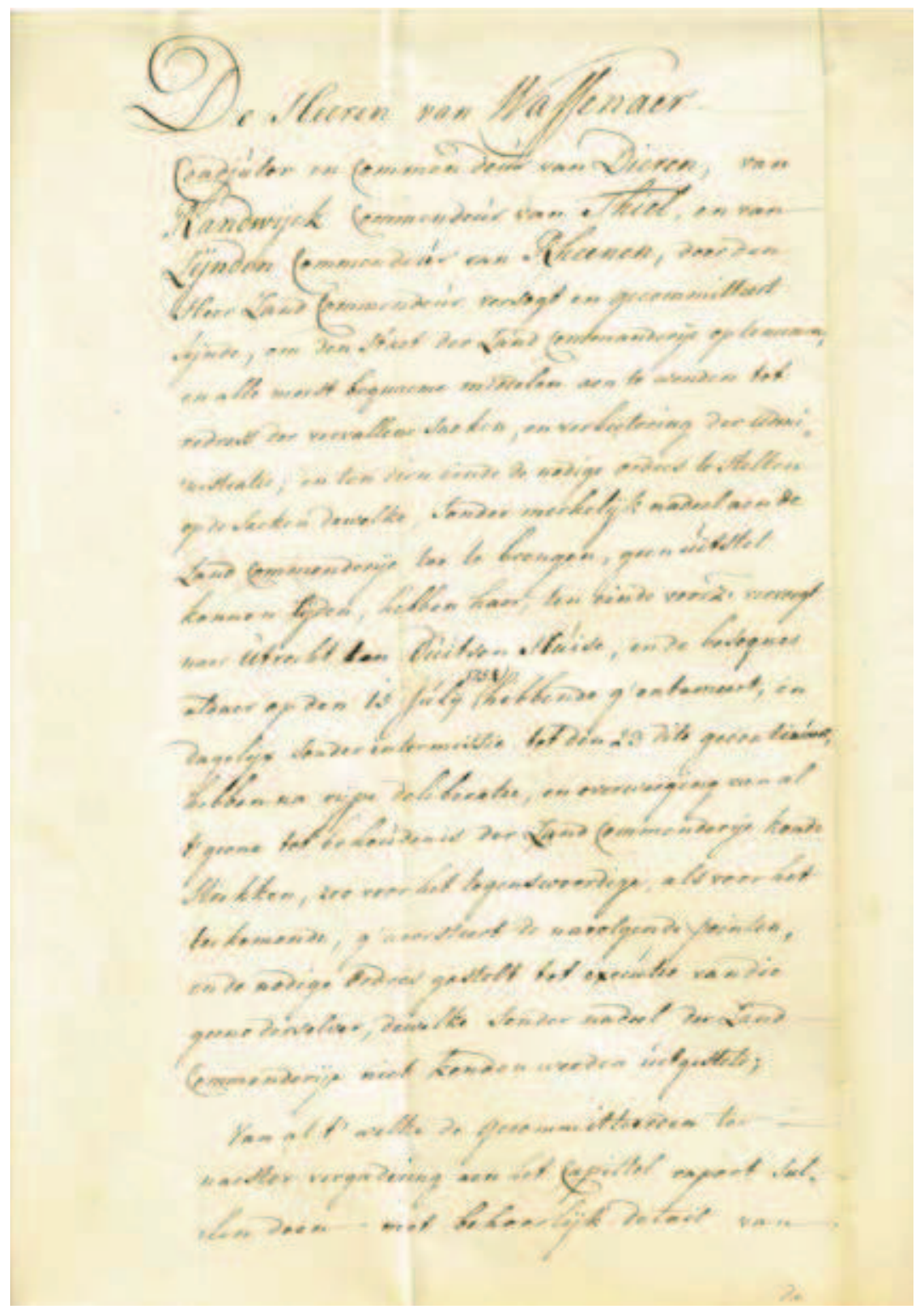

Illustration 1 


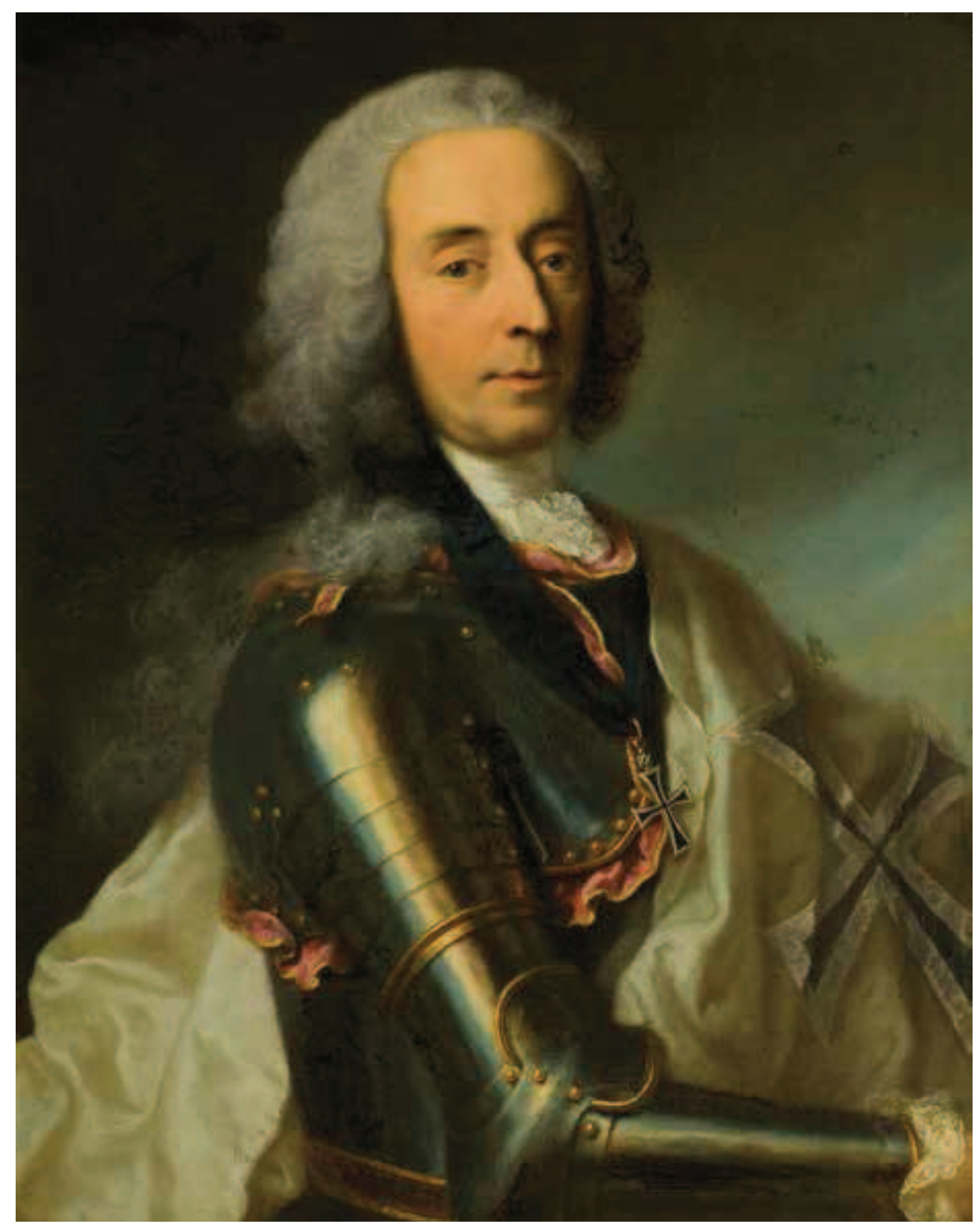

Illustration 2 


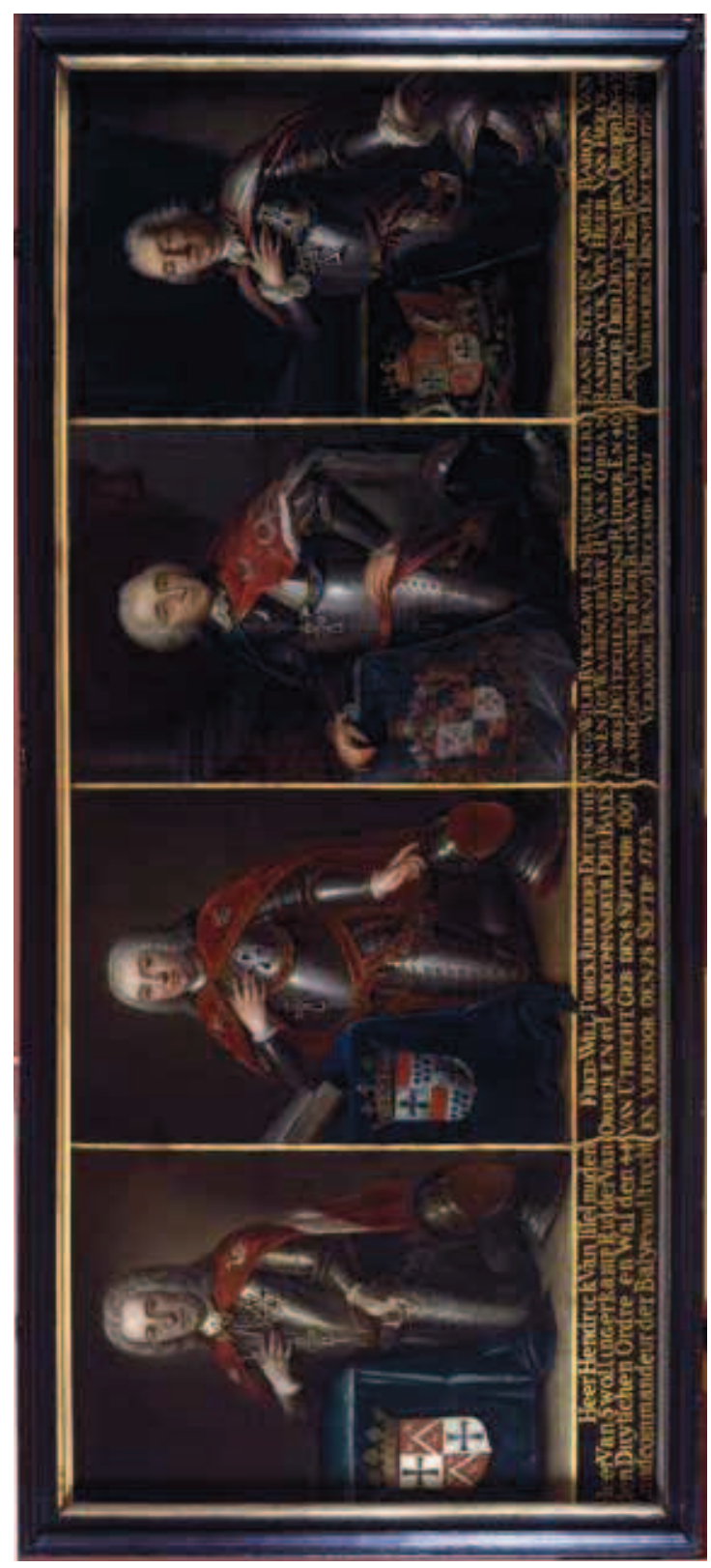

Illustration 3 


\section{SOURCES AND Literature}

Aalbers, Johan. "Geboorte en geld. Adel in Gelderland, Utrecht en Holland tijdens de eerste helft van de achttiende eeuw." In De bloem der natie. Adel en patriciaat in de Noordelijke Nederlanden, edited by Johan Aalbers and Maarten Prak, 56-78. Meppel: Boom Meppel, 1987.

Aalbers, Johan, "Rond een bezending naar Munsterland." In Unico Wilhelm van Wassenaer 1692-1766. Componist en staatsman, edited by Rudolf Rasch and Kees Vlaardingerbroek, $197-2$ I 0 . Zutphen: Centrum Nederlandse Muziek, 1993.

Arnold, Udo., Bott, Gerhard., red. 8oo Jahre Deutscher Orden. Ausstellung des Germanischen Nationalmuseums Nürnberg in Zusammenarbeit mit der Internationalen Historischen Kommission zur Erforschung des Deutschen Ordens, Germanisches Nationalmuseum, 30.6.-30.9.199o. Gütersloh, München: Bertelsmann Lexikon 1990.

Bootsma, Nicolaas A. De Hertog van Brunswijk I750-1759. Assen: Prakke \& Prakke, 1962. Brink, Wilma van den. "Utrechtse adellijke vrouwen en prebenden in de achttiende eeuw." Virtus. Jaarboek voor Adelsgeschiedenis I I (2004): 43-53.

Bruin, Renger E. de. Bedreigd door Napoleon. De Ridderlijke Duitsche Orde, Balije van

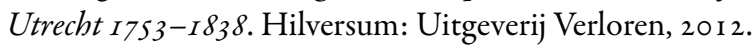

Bruin, Renger E. de. "Hidden in the Bushes: The Teutonic Order of the Bailiwick of Utrecht in the 1780-1 806 Revolutionary Period." In The Military Orders. Vol. 5, edited by Peter Edbury. 349-36r. Farnham: Ashgate, 2012.

Bruin, Renger E. de. "De Ridderlijke Duitse Orde Balije van Utrecht, een adellijk distinctie-instrument in de Republiek der Verenigde Nederlanden gedurende de achttiende eeuw." Virtus. Jaarboek voor Adelsgeschiedenis 16 (2009): 34-53.

Bruin, Renger E. de. "Utrechter Patrizier und Bürger als Gutsbesitzer. Ein Aristokratisierungsprozess, 1600-1850." In Städtische Siedlungen und ihr Umland. Siedlungsforschung, Archäologie - Geschichte - Geographie. Vol. 26, edited by Johannes Renes and Winfried Schenk, 87-126. Bonn: Arkum, 2008.

Bruin, Renger E. de. We and Our Successors Shall Do Justice by All. Provincial Government in Utrecht: A Historical Perspetive. Utrecht: Centraal Museum, 2003.

Brusse, Paul., Mijnhardt, Wijnhardt W. “Towards a new template for Dutch History." Virtus. Yearbook of the History of the Nobility 19 (20 I 2): 173- I 80.

Demel, Bernhard. "Die Deutschordensballei Utrecht in der Reichs- und Ordensüberlieferung von der frühen Neuzeit bis in die Zeit Napoleons." In Unbekannte Aspekte der Geschichte des Deutschen Ordens, edited by Bernhard Demel, 9-122. Wien, Köln, Weimar: Böhlau, 2005.

Faber, Johannes A. "Cattle Plague in the Netherlands during the Eigteenth Century." Mededelingen van de Landbouwhogeschool te Wageningen 62 (1962): I-7.

Grögor-Schiemann, Daniela. Die Deutschordensballei Utrecht während der Reformationszeit: die Landkommende zwischen Rebellion und Staatsbildung. Weimar: VDG, 2015. 
Israel, Jonathan I. The Dutch Republic. Its Rise, Greatness and Fall, 1477-1806. Oxford: Clarendon Press, I 995.

Kuiper, Yme. Adel in Friesland 1780-1880. Groningen: Wolters Noordhoff, I 990.

Lademacher, Horst. Geschichte der Niederlande. Politik, Verfassung, Wirtschaft. Darmstadt: Wissenschaftliche Buchgesellschaft, 1983.

Meuwissen, Daantje. Gekoesterde traditie. De portretreeks met de landcommandeurs van de Utrechtse Balije van de Ridderlijke Duitsche Orde. Hilversum: Uitgeverij Verloren, $20 \mathrm{II}$.

Mijnhardt, Wijnhardt W. "The Dutch Enlightenment (1650-1800): problems and definitions." In Centres et péripheries: Les Lumières de Belfast à Bejing, edited by Marie-Christine Skuncke, I 70-1 83. Paris: Champion, 2003.

Mijnhardt, Wijnhardt W. "Urbanization, Culture and the Dutch Origins of the European Enlightenment." BMGN The Low Countries Historical Review I 25 (2010): I 4 I - 178.

Mol, Johannes A. "The 'Hospice of the German Nobility': Changes in the Admission Policy of the Teutonic Knights in the Fifteenth Century." In Mendicants, Military Orders and Regionalism in Medieval Europe, edited by Jürgen Sarnowsky, I I 5-1 30 . Aldershot: Ashgate, I999.

Mol, Johannes A. "Trying to survive. The Military Orders in Utrecht, I $580-1620$." In The Military Orders and the Reformation. Choices, State Building and the Weight of Tradition, edited by Johannes A. Mol, Klaus Militzer and Hellen Nicholson, i 8 I-208. Hilversum: Uitgeverij Verloren, 2006.

Mol, Johannes A. "Crisis in Pruisen, crisis in de balijen? De casus Utrecht, I 443-1 469." In Vechten, bidden en verplegen. Opstellen over de ridderorden in de Noordelijke Nederlanden, edited by Johannes A. Mol, 206-222. Hilversum: Uitgeverij Verloren, 20 I I.

Price, John L. Culture and Society in the Dutch Republic during the I th Century. London: Harper Collins, I 974

Pörnbacher, Karl. "Daß die Untertanen nach aller Gerechtigkeit geführt und regiert werden. Crescentia Höß von Kaufbeuren als geistliche Beraterin des Kurfürsten Clemens August." In Der Riss im Himmel. Clemens August und seine Epoche, edited by Frank G. Zehnder and Werner Schäfke. Vol. 5: Hirt und Herde. Religiosität und Frömmigkeit im Rheinland des I 8. Jahrhunderts, I 97-2 I 2. Köln: DuMont, 2000.

Rasch, Rudolf., Vlaardingerbroek, Kees., edit. Unico Wilhelm van Wassenaer I692-1766, componist en staatsman. Zutphen: Centrum Nederlandse Muziek, I 993.

Rommes, Ronald N. J. “'Geen vrolyk geloei der melkzwaare koeien'. Runderpest in Utrecht in de achttiende eeuw." Jaarboek Oud-Utrecht (200 I): 87- I 36.

Sijs, Nicoline van der. "De geschiedenis van de Nederlandse taal." In Dialectatlas van het Nederlands, edited by Nicoline van der Sijs, 29-84. Amsterdam: Uitgeverij Bert Bakker, $20 \mathrm{I}$ I.

Streng, Jean. "De adel in de Republiek." Virtus. Jaarboek voor Adelsgeschiedenis 10 (2003): $7 \mathrm{I}-\mathrm{IOI}$ 
Vey, Jan H. Mestdagh de. De Utrechtse Balije der Duitse Orde. Ruim 750 jaar geschiedenis van de Orde in de Nederlanden. Utrecht, Bilzen: S. Vanaudenhove \& P. A. baron van der Borch, I 989.

Vries, Jan de. Woude, Ad van der. The First Modern Economy: Success, Failure, and Perseverance of the Dutch Economy, I50o-I8I5. Cambridge: Cambridge University Press, I 997.

\section{Abstract \\ A successful reorganization. The Teutonic Order Bailiwick of Utrecht, 1753-1795}

In 1753 the newly elected coadjutor of the Teutonic Order Bailiwick of Utrecht, Unico Wilhelm Count van Wassenaer van Twickel, analyzed the state of the order, being a total chaos after a long period of decay. After he was commissioned by the other members to make a plan for improvement, Van Wassenaer managed to implement a reorganization that was highly successful. It was based on a rational management of the possessions by a professional steward, concentration of supervision at the land commanderie in Utrecht, a reduced role of the members and automatic succession of the land commander after his death by the coadjutor to prevent an interregnum as between 1751 and 1753 . According to new rules, Van Wassenaer became land commander in 1761 and finished the reorganization during his time in office. After his death in 1766 his successor followed his course. The competent steward Gijsbert Dirk Cazius managed to turn the deficits into increasing surpluses, making the Baliwick of Utrecht an organization that was financially robust by the time it was threatened by the French Revolution. In the success of his financial policy Cazius, who worked closely together with the land commanders he served, was helped by the economic developments: the rising prices of agricultural products enabled farmers to pay higher rents that were collected punctually by the steward. In its rational and utilitarian character the reorganization seems to breeze the spirit of the Enlightenment, but the emphasis on noble ancestors and Reformed confession as prerequisites for membership make the Bailiwick of Utrecht a typical institution of the Ancien Regime. However, the realized plans of Van Wassenaer led the Baliwick out of a desperate state and played a crucial role in the survival to the present day. 